\title{
Under Pressure: Mechanochemical Effects on Structure and Ion Conduction in the Sodium-Ion Solid Electrolyte $\mathrm{Na}_{3} \mathrm{PS}_{4}$
}

\begin{abstract}
Theodosios Famprikis ${ }^{a, b, k,+, \neq *}$, Ulas Kudu ${ }^{a}$, James A. Dawson ${ }^{b, c}$, Pieremanuele Canepa ${ }^{d}$, François Fauth ${ }^{e}$, Emmanuelle Suard ${ }^{f}$, Mohamed Zbirif ${ }^{f}$ Damien Dambournet ${ }^{g, \neq}$, OlafJ. Borkiewicz $^{h}$, Houssny Bouyanfif ${ }^{i}$, Steffen P. Emge ${ }^{j}$, Sorina Cretu ${ }^{a}$, Jean-Noël Chotard ${ }^{a, l}$, Clare P. Grey ${ }^{j,+}$, Wolfgang G. Zeier ${ }^{k}$, M. Saiful Islam ${ }^{b,+*}$ and Christian Masquelier ${ }^{a,+, * *}$
\end{abstract}

${ }^{a}$ Laboratoire de Réactivité et Chimie des Solides (LRCS), CNRS UMR 7314, Université de Picardie Jules Verne, 80039 Amiens, France

${ }^{b}$ Department of Chemistry, University of Bath, BA2 7AY, United Kingdom

${ }^{c}$ Chemistry-School of Natural and Environmental Sciences, Newcastle University, Newcastle upon Tyne NE1 7RU, United Kingdom

${ }^{\mathrm{d} D e p a r t m e n t ~ o f ~ M a t e r i a l s ~ S c i e n c e ~ a n d ~ E n g i n e e r i n g, ~ T h e ~ N a t i o n a l ~ U n i v e r s i t y ~ o f ~ S i n g a p o r e, ~ 117576, ~ S i n g a p o r e ~}$ ${ }^{e}$ CELLS - ALBA Synchrotron, Cerdanyola del Vallès, o8290 Barcelona, Spain ILL

fInstitut Laue-Langevin (ILL), BP 156, 71 Avenue des Martyrs, 38042 Grenoble, France

gPhysico-Chimie des Electrolytes et Nano-systèmes Interfaciaux (PHENIX), CNRS UMR 8234, Sorbonne Université, F-75005 Paris, France

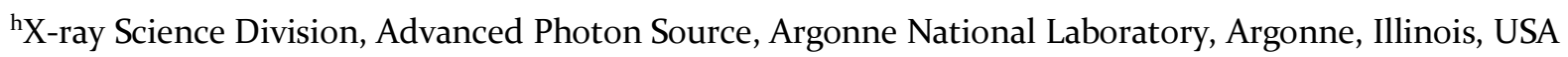

iLaboratoire de Physique de la Matière Condensée (LPMC), UR 2081, Université de Picardie Jules Verne, Amiens 80039, France

¡Department of Chemistry, University of Cambridge, CB2 1EW, United Kingdom

kInstitute of Physical Chemistry, Justus-Liebig-University Giessen, Heinrich-Buff-Ring 17, D-35392 Giessen, Germany

${ }^{\dagger}$ ALISTORE European Research Institute, CNRS FR 3104, 80039 Amiens, France

${ }^{\ddagger}$ Réseau sur le Stockage Électrochimique de l’Énergie (RS2E), CNRS FR 3459, 80039 Amiens, France

Corresponding Authors

theo.famprikis@u-picardie.fr; m.s.islam@bath.ac.uk; christian.masquelier@u-picardie.fr 
ABSTRACT: Fast-ion conductors are critical to the development of solid-state batteries. The effects of mechanochemical synthesis that lead to increased ionic conductivity in an archetypical sodium-ion conductor $\mathrm{Na}_{3} \mathrm{PS}_{4}$ are not fully understood. We present here a comprehensive analysis based on diffraction (Bragg and pair distribution function), spectroscopy (impedance, Raman, NMR and INS) and ab-initio simulations aimed at elucidating the synthesis-property relationships in $\mathrm{Na}_{3} \mathrm{PS}_{4}$. We consolidate previously reported interpretations regarding the local structure of ball-milled samples, underlining the sodium disorder and showing that a local tetragonal framework more accurately describes the structure than the originally proposed cubic one. Through variable-pressure impedance spectroscopy measurements, we report for the first time the activation volume for $\mathrm{Na}^{+}$migration in $\mathrm{Na}_{3} \mathrm{PS}_{4}$, which is $\sim 30 \%$ higher for the ballmilled samples. Moreover, we show that the effect of ball-milling on increasing the ionic conductivity of $\mathrm{Na}_{3} \mathrm{PS}_{4}$ to $\sim 10^{-4} \mathrm{~S} / \mathrm{cm}$ can be reproduced by applying external pressure on a sample from conventional high temperature ceramic synthesis. We conclude that the key effects of mechanochemical synthesis on the properties of solid electrolytes can be analyzed and understood in terms of pressure, strain and activation volume. 


\section{INTRODUCTION}

$\mathrm{Na}_{3} \mathrm{PS}_{4}$ is an archetypical $\mathrm{Na}^{+}$ion conductor in the thiophosphate family, and of particular interest for the development of sodium solid-state batteries (SSBs). A tetragonal phase at room temperature $\left(\alpha, P \overline{4} 2_{1} c\right)$ and a closely related cubic phase at $\mathrm{T}>250{ }^{\circ} \mathrm{C}(\beta$, $I \overline{4} 3 \mathrm{~m}$ ) have been described in earlier studies ${ }^{1}$ and we recently reported the existence of a third polymorph $(\gamma, F d d d)$ with plastic crystal characteristics ${ }^{2} \cdot \mathrm{Na}_{3} \mathrm{PS}_{4}$ synthesized through conventional high temperature solid-state routes typically exhibits ionic conductivities of the order of $10^{-5} \mathrm{~S} / \mathrm{cm}$, which is somewhat low for SSB applications ${ }^{3}$. A resurgence of interest in $\mathrm{Na}_{3} \mathrm{PS}_{4}$ had been induced by Hayashi et al. who showed that the mechanochemical synthesis route (i.e. ball-milling) can lead to increased room temperature ionic conductivities of the order of $10^{-4} \mathrm{~S} / \mathrm{cm}$, which allowed its integration into laboratory-scale SSB prototypes ${ }^{4-6}$. They proposed that this effect was due to the stabilization of the cubic $\beta$-phase as a result of ball-milling.

There is a discrepancy in the literature regarding the exact average structure $\beta-\mathrm{Na}_{3} \mathrm{PS}_{4}$, with three different models proposed from the analysis of $x$-ray diffractograms ${ }^{7-9}$. The difference between these models pertains to the Na position(s) which have been described either by a fully occupied $6 \mathrm{~b}$ site 7 , a $25 \%$ occupied $24 \mathrm{f}$ site ${ }^{8}$ or a combination of partially occupied $6 \mathrm{~b}$ and $12 \mathrm{~d}$ sites ${ }^{9}$. Considering the structure as an ionic-molecular crystal of $\mathrm{Na}^{+}$ and $\mathrm{PS}_{4}{ }^{3-}$, it can be described as a body-centered arrangement of the $\mathrm{PS}_{4}{ }^{3-}$ anions (corners and center of the cell) in which all six-coordinated "interstitial" sites (centers of faces and edges) are occupied by $\mathrm{Na}^{+}$( similarly to the intermetallic $\mathrm{AuSb}_{3}{ }^{10}$ ). In this view, the latter "interstitial" sites for $\mathrm{Na}^{+}$are described by the $6 \mathrm{~b}$ position of the $I \overline{4} 3 \mathrm{~m}$ space group. The $12 \mathrm{~d}$ and $24 \mathrm{f}$ sites then bridge the $6 \mathrm{~b}$ sites and their partial occupation can be interpreted as a sign of the $\mathrm{Na}^{+}$diffusivity in the structure, tracing the diffusion pathways which are parallel to the lattice vectors a, b and c (Figure 1). The $12 \mathrm{~d}$ site in particular is located at the midpoint between stable $6 \mathrm{~b}$ sites and is considered the "transition" site in the diffusion pathway,"11. 


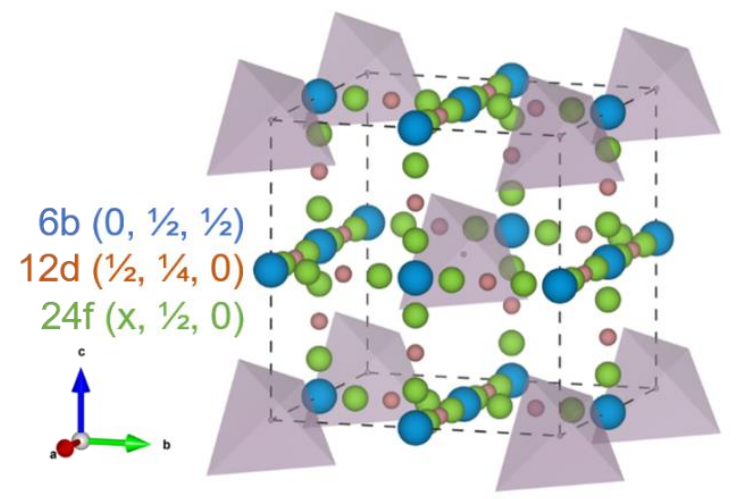

Figure 1: Distribution of $\mathrm{Na}^{+}$in $\beta-\mathrm{Na}_{3} \mathrm{PS}_{4}(\mathrm{I} \overline{4} 3 \mathrm{~m})$. $\mathrm{PS}_{4}{ }^{3-}$ moieties represented by purple tetrahedra and proposed $\mathrm{Na}^{+}$positions represented by spheres colored according to their respective Wyckoff site.

Nevertheless, the original hypothesis of the crystal structure ( $\alpha$ vs. $\beta$ ) decisively affecting ion conduction ${ }^{4,5}$ has recently been challenged both experimentally and computationally: ab initio molecular dynamics simulations show similar diffusivity for $\mathrm{Na}^{+}$in both phases ${ }^{1{ }^{-}}$ 13 and pair distribution function (PDF) analyses reveal an identical local structure regardless of the sample preparation method 7. We note that Hayashi et al. described the material in their related patent as "contain[ing] tetragonal $\mathrm{Na}_{3} \mathrm{PS}_{4}$ "14. Recent studies have also demonstrated tetragonal samples with conductivities up to $10^{-3} \mathrm{~S} / \mathrm{cm}$ through quenching and/or annealing of ball-milled samples ${ }^{7,15}$.

The conductivity-enhancing effect of mechanochemistry is quite reproducible in $\mathrm{Na}_{3} \mathrm{PS}_{4}$ 16 and similar observations have been reported for several mechanically soft solidelectrolytes (e.g. $\mathrm{Li}_{3} \mathrm{PS}_{4},{ }^{17} \mathrm{LiBH}_{4},{ }^{18} \mathrm{Ag}_{3} \mathrm{PS}_{4}{ }^{19}, \mathrm{Na}_{11} \mathrm{Sn}_{2} \mathrm{PSe}_{12}{ }^{20}$ and $\mathrm{LiYCl}_{6}{ }^{21}$ ). However, the effect of mechanochemistry to produce highly conducting solid electrolytes remains poorly understood. Multiple hypotheses have been proposed including atomic-scale changes in crystal structure and/or in point defect concentrations, microstructural effects (such as grain boundaries), as well as mesostructural parameters such as particle size/shape and associated surface effects ${ }^{18,22,23}$. Both simulations and experiments show that introducing sodium ion defects through aliovalent doping can decisively enhance ion-transport in $\mathrm{Na}_{3} \mathrm{PS}_{4}{ }_{9,11,13,24-30}$. Through novel atomistic simulations, the effect of grain boundaries on ion 
conduction in $\beta-\mathrm{Na}_{3} \mathrm{PS}_{4}$ has recently been examined ${ }^{31}$, showing that ion transport is not significantly affected by grain boundaries in this material in contrast to sodium phosphate analogues.

Ball-milling synthesis consists in carrying out chemical reactions by application of mechanical force (mechanochemistry) in the form of collisions between the accelerated milling media (balls) and reagents. In contrast to high temperature ceramic synthesis where the energy input is thermal, in mechanochemical approaches the energy input is mechanical, i.e., pressure. Once an exothermic reaction is mechanically activated, the heat generated can sustain the reaction (in which case it can be termed a mechanically-induced self-sustaining reaction, MSR ${ }^{2-34}$ ). The continually applied mechanical input can have side-effects on the resulting products, such as the reduction of particle- and crystallite- size as well as the accumulation of micro- and macro-strain. Despite the wide-spread use of ball-milling for the synthesis of soft solid electrolytes for batteries, the effects of particle size and strain on relevant functional properties have hardly been the subject of detailed investigation.

In this study, we explored the hypothesis of "mechanical" effects being the origin of the anomalously high conductivity of ball-milled $\mathrm{Na}_{3} \mathrm{PS}_{4}$ and related systems. We use this generic umbrella term to include effects arising from the application of mechanical pressure (including mechanochemical synthesis) and capture the following features:

(a) Micro- and macrostrain on the local- and average scales, respectively, which can dramatically affect the volume available for $\mathrm{Na}^{+}$diffusion and correlate with the activation volumes measured by pressure-dependent conductivity measurements.

(b) Effective interparticle contact which determines ionic percolation through solid electrolyte particles in a macroscopic pellet and can severely affect the measured ionic conductivity.

(c) Possible pressure-induced structural phase transitions. 
Using a powerful combination of diffraction (Bragg and PDF), spectroscopic (impedance, Raman, inelastic neutron and nuclear magnetic resonance) and ab initio simulation techniques, we show that the main difference imparted on $\mathrm{Na}_{3} \mathrm{PS}_{4}$ by mechanochemistry is a distinct accumulated strain both at the local and average scales. This strain affects the dependence of the ionic conductivity on the applied pressure as quantified by a higher activation volume. Finally, we demonstrate that a key component to the conductivity increase ascribed to mechanochemistry, is due to a mesostructural consolidation effect, increasing the effective interparticle contact area, which can be reproduced by a simple application of external pressure on a conventional hightemperature-synthesized sample.

\section{RESULTS AND DISCUSSION}

In the majority of our study we compare two samples: a ground-state tetragonal $\alpha-\mathrm{Na}_{3} \mathrm{PS}_{4}$ prepared by high-temperature solid-state synthesis (HT-tet- $\mathrm{Na}_{3} \mathrm{PS}_{4}$ ) and a metastable cubic $\beta-\mathrm{Na}_{3} \mathrm{PS}_{4}$ prepared by ball-milling (BM-cub-Na $\mathrm{PS}_{4}$ ). Inspired by previous work 7,15 we also synthesized an "intermediate" sample prepared by rapid high temperature annealing of a ball-milled sample, also resulting in tetragonal long-range symmetry $\left(\mathrm{BM}\right.$-tet- $\left.\mathrm{Na}_{3} \mathrm{PS}_{4}\right)$; which we invoke in certain parts of this paper. In agreement with previous studies, the ball-milled samples exhibit significantly higher room-temperature ionic conductivity (BM-tet and $\mathrm{BM}-\mathrm{cub}, \sim 10^{-4} \mathrm{~S} / \mathrm{cm}$ ) 4-7,15,16 compared to the hightemperature-synthesized material (HT-tet, $\sim 10^{-5} \mathrm{~S} / \mathrm{cm}$ ) 1,7,11,12,15.

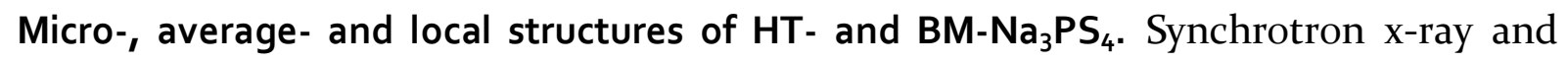
neutron diffractograms confirm the purity of the products and the crystal structures can be accurately refined (Figs. S1-S3) using starting models of Jansen and Henseler ${ }^{1}$ for the tetragonal $\alpha$-phase and of Krauskopf et al. 7 for the cubic $\beta$-phase, respectively. The two phases are very closely related, with the phase transition involving the convergence of the lattice parameters, a slight orientational tilt of the $\mathrm{PS}_{4}{ }^{3-}$ tetrahedra and slight displacements of $\mathrm{Na}^{+}$to the middle of the edges and faces of the cubic cell. The close group-subgroup relationship between the two polymorphs 35 (Table $\mathrm{S}_{4}$ ) is evident in the diffractograms 
(Figure 2), which exhibit very similar features with characteristic merging and extinction of peaks from the tetragonal to the cubic phase.

From careful analysis of the x-ray Bragg diffractograms we aimed at extracting microstructural parameters of the sample powders. Finite crystallite size and micro-strain both cause peak broadening, which can be differentiated and quantified by analyzing the peak shapes (Lorentzian vs. Gaussian) and widths as a function of the scattering vector (i.e. 2 $\theta$ ). The associated Williamson-Hall plot is given in SI Figure $S_{4}$ Here, crystallite size refers to the average distance between coherent crystallographic domains in the powder, and micro-strain refers to variation in the lattice parameters between such individual domains.
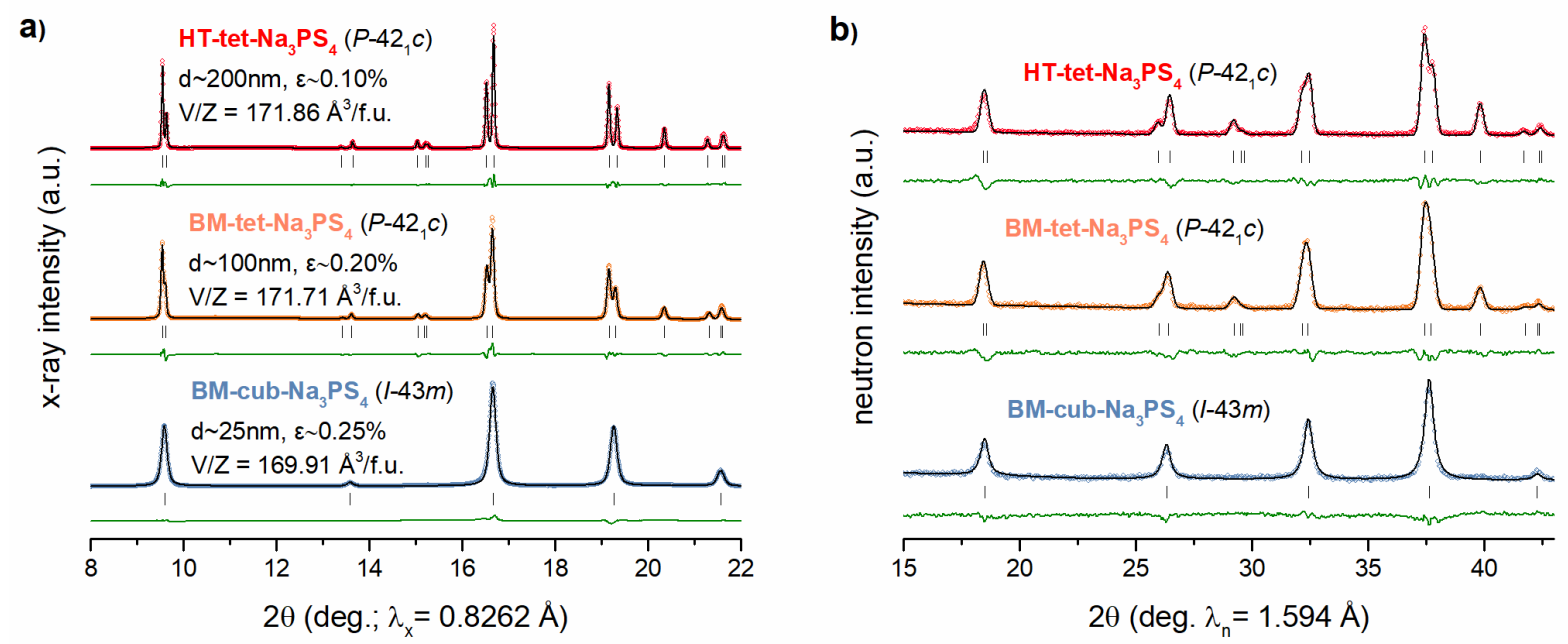

Figure 2 [Double Column]: Sections of the synchrotron $x$-ray (a) and neutron (b) diffractograms of the $\mathrm{Na}_{3} \mathrm{PS}_{4}$ samples with associated Rietveld refinements. $d$ and $\varepsilon$ are the isotropic crystallite size and average microstrain, respectively, extracted from analysis of the peak shapes of the $x$-ray datasets. Both panels compare the same q-range.

Figure 1 shows distinct peak broadening going from HT-tet to BM-tet to BM-cub, which we quantified as both a decrease in crystallite size and an increase in micro-strain. The HT-tet sample shows a large crystallite size of $\sim 200 \mathrm{~nm}$ with negligible average micro-strain of $\sim 0.1 \%$, while the analysis of the BM-cub sample results in a minimal grain size of $\sim 25 \mathrm{~nm}$ 
with significant micro-strain of $\sim 0.25 \%$. These microstructural differences can be attributed to the different synthesis routes: high-temperature annealing results in grain growth and strain release, while high-energy ball-milling subjects the sample to sizable mechanical forces and frequent impact, resulting in crystallite fracture and strain accumulation. It is interesting to note that the "intermediate" sample BM-tet exhibits a larger crystallite size ( $\sim 100 \mathrm{~nm})$ but rather high micro-strain ( 0.2\%, similar to BM-cub). We conclude here that the rapid 2o-minute annealing of the ball-milled sample leads to some grain-growth but is not sufficient to significantly relieve the micro-strain induced by ball-milling. We attempted to corroborate these results by transmission electron microscopy but were impeded by the well-known instability of thiophosphates under the high electron doses required.

In addition, the observed micro-strain seems to also be associated with compressive macro-strain, i.e., a shrinkage of the average lattice volume observable from diffraction peak positions. An inspection of the superimposed PDFs of the samples (Figures $\mathrm{S}_{5}$ ) and their refinements (Figs S6-S8, extracted by total scattering experiments, is consistent with the Bragg diffractograms: the two tetragonal samples (HT-tet, BM-tet, indexed in $P \overline{4} 2_{1} c$ ) largely coincide; while the BM-cub (indexed in $I \overline{4} 3 \mathrm{~m}$ ) shows distinct deviations and shifts to lower interatomic correlation distance, which indicates the shrinkage of the cell. From refinements of the Bragg and PDF datasets, the lattice volume decreases from 171.9 (172.7) to $171.7(172.3)$ to $169.9(170.7) \AA^{3}$ per formula unit from HT-tet- to BM-tet- to BM-cub- $\mathrm{Na}_{3} \mathrm{PS}_{4}$, respectively. We note that volume variations could be indicative of variations in the mobile defect concentrations as $\mathrm{Na}$ vacancies are expected to cause local contraction of the lattice. We conclude that the mechanical energy input during the ballmilling synthesis reduces the particle and crystallite size of the resulting powder as well as imparts a micro- and macro-strain on the structure of the resulting crystal.

In order to explain the stabilization of the average cubic structure after ball-milling, we have investigated the relative phase stability of the $\alpha$ - and $\beta$-polymorphs as a function of pressure using density functional theory (DFT) calculations. Consistent with experimental 
observation, the cubic $\beta$-polymorph has a higher formation energy and smaller equilibrium volume (Figure S9 and Table Si1) than the tetragonal $\alpha$-phase in the absence of applied pressure. Using the DFT-calculated values for energy $(\mathrm{E})$, volume $(\mathrm{V})$ and pressure $(\mathrm{P})$ we calculated the enthalpy of formation $(\mathrm{H}=\mathrm{E}+\mathrm{PV})$ for each polymorph as the applied pressure is varied. As the pressure increases, the lattice parameters of the tetragonal phase converge to the same value and the absolute enthalpy difference between the two polymorphs decreases, indicating a stabilization of the cubic polymorph (Figure 3). The enthalpy difference approaches zero in the range $10-15 \mathrm{GPa}$ and as such a pressure-induced phase transition from tetragonal to cubic is predicted in this pressure range. We conclude that the stabilization of the cubic $\beta$-polymorph for the BM-cub sample can be understood as a result of the strain imparted on the sample during the mechanochemical synthesis. In-situ variable-pressure diffraction experiments are scheduled to experimentally confirm this computationally-predicted pressure-induced phase transition of $\mathrm{Na}_{3} \mathrm{PS}_{4}$.

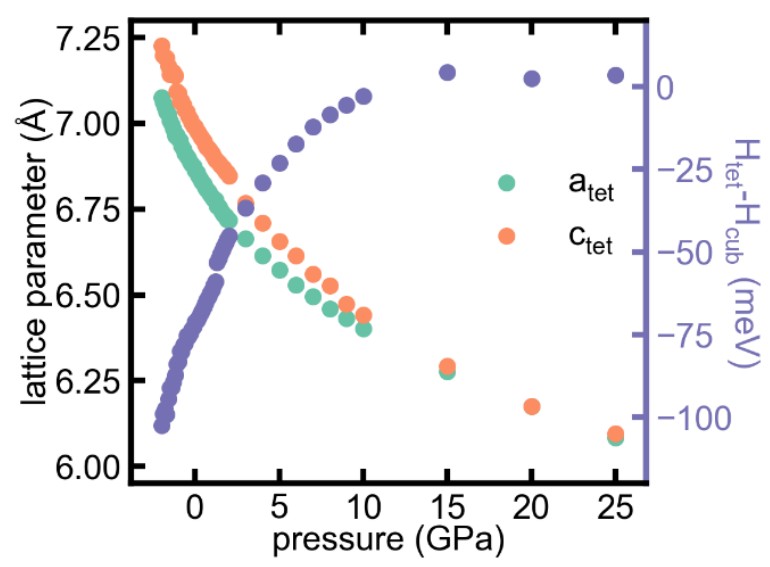

Figure 3 [single column]: Variation of the calculated lattice parameters of $\alpha-\mathrm{Na}_{3} \mathrm{PS}_{4}$ (green and orange) and the difference in enthalpy (in violet) between $\alpha$ - and $\beta-\mathrm{Na}_{3} \mathrm{PS}_{4}$ as a function of pressure.

Next, we utilized Rietveld-type refinements of our diffraction data to probe the precise $\mathrm{Na}$-ion distribution in $\mathrm{BM}$-cub- $\mathrm{Na}_{3} \mathrm{PS}_{4}$. Variations in the possible sodium positions (see 
Figure 1) yield small differences of the peak intensities of Bragg diffractograms, which is why we complemented this analysis with total scattering. On this basis, we have attempted numerous fits of our three diffractograms of BM-cub- $\mathrm{Na}_{3} \mathrm{PS}_{4}$ (x-ray Bragg, neutron Bragg and $x$-ray total scattering) in inverse- and direct-space (Rietveld and PDF, respectively) in the cubic $I \overline{4} 3 \mathrm{~m}$ framework in an effort to resolve the controversy. The results are tabulated in Table S12 and rationalized below. We find that the majority of Na scattering density is centered around the $6 \mathrm{~b}$ site, but appears to be non-spherical, probably owing to the directionality of $\mathrm{Na}^{+}$conduction through the lattice. Splitting the $6 \mathrm{~b}$ site into a partially filled $24 \mathrm{f}$ site (with $\mathrm{x}$ close to 0.5 ) helps to better capture this anisotropy as does fitting anisotropic (ellipsoidal) thermal displacement parameters. Neither inverse- nor directspace analysis reveal signs of significant occupation of the $12 \mathrm{~d}$ "transition" site alone or in partial occupation, in agreement with the conclusions of Krauskopf et al. 7 The model that best reconciles all diffraction data sets is one with a $25 \%$-occupied $24 \mathrm{f}$ site $(x \approx 0.05)$ with anisotropic displacement parameters. This is in good agreement with the interpretation of Nishimura et al. for a $\beta-\mathrm{Na}_{3} \mathrm{PS}_{4}$ sample measured at $600 \mathrm{~K}^{8}$, and indicative of directional mobility of $\mathrm{Na}^{+}$in this structure. 
a)

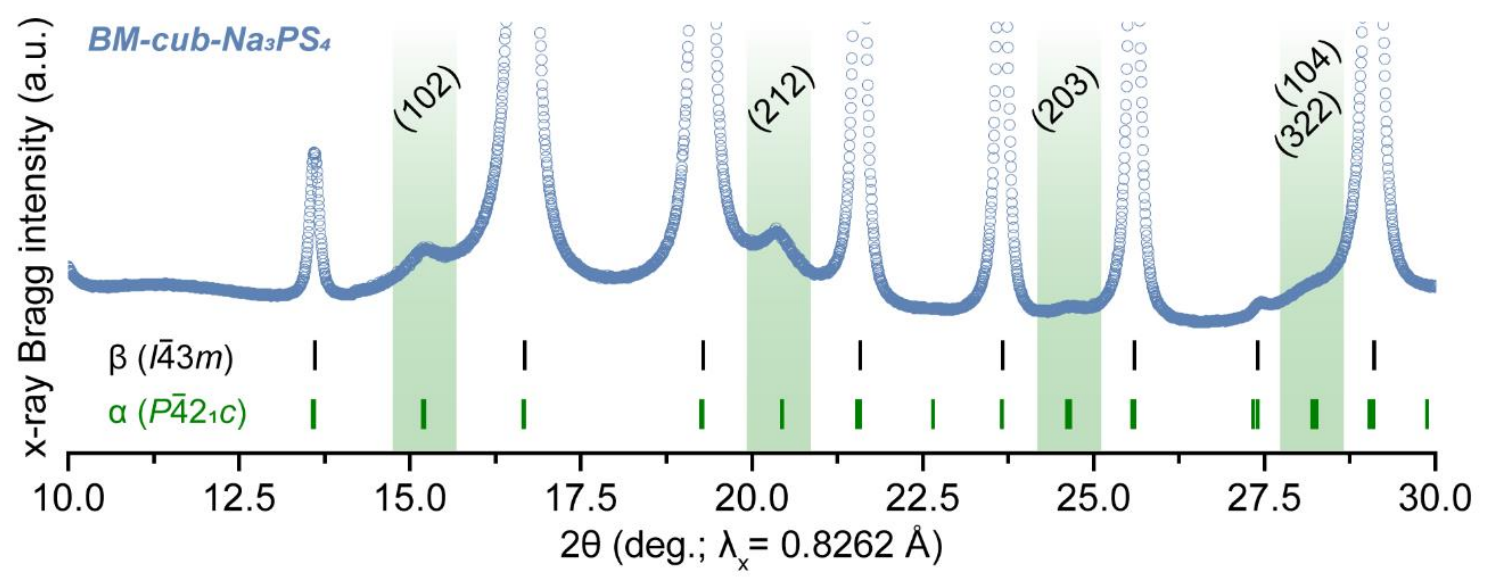

b)

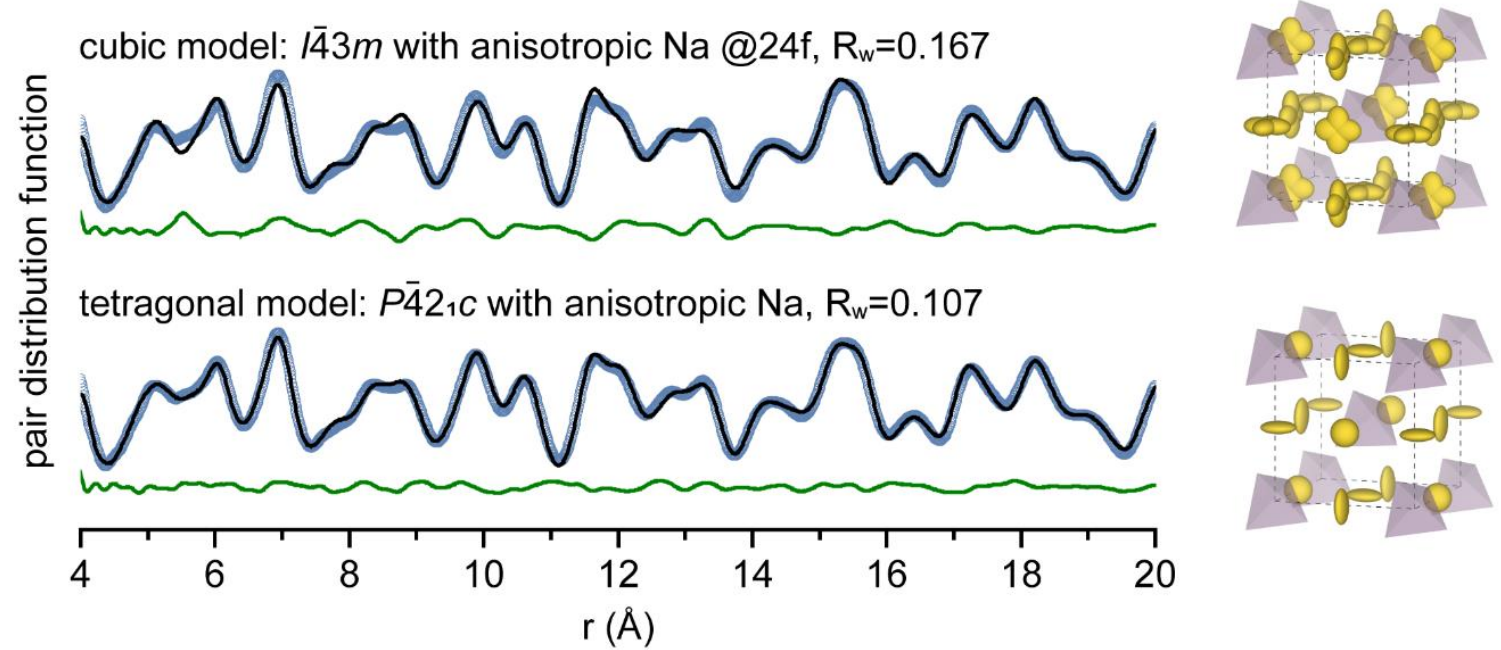

Figure 4 [Double Column]. (a) Highlight of the weak unindexed "tetragonal" peaks in the Bragg diffractogram of BM-cub-Na $\mathrm{PS}_{4}$ (b) Comparison of the PDF fits of $\mathrm{BM}$-cub- $\mathrm{Na}_{3} \mathrm{PS}_{4}$ using cubic and tetragonal average symmetry with visualizations of the resulting structural models. Refinements performed for a symmetry-confined, single-unit-cell in the range 4-20 A.

In contrast to their interpretation from x-ray Bragg diffraction 4,5, Hayashi et al., following their seminal report on the mechanochemical synthesis of "cubic" $\mathrm{Na}_{3} \mathrm{PS}_{4}$, filed an associated patent describing the material as "containing tetragonal $\mathrm{Na}_{3} \mathrm{PS}_{4}{ }^{14}$, without, however, further substantiation of that description. A close inspection of the synchrotron $\mathrm{x}$-ray diffractogram of $\mathrm{BM}$-cub- $\mathrm{Na}_{3} \mathrm{PS}_{4}$ (Figure 4a) reveals weak, broad peaks, unindexed by the cubic $I \overline{4} 3 \mathrm{~m}$ space group of $\beta-\mathrm{Na}_{3} \mathrm{PS}_{4}$, but which correspond well to the expected peaks for the tetragonal $P \overline{4} 2_{1} c$ space group of $\alpha-\mathrm{Na}_{3} \mathrm{PS}_{4}$ for $\mathrm{a} \approx \mathrm{c}$. In particular, the (102), (212), 
(203), (322) and (104) families of peaks of $P \overline{4} 2_{1} c$ are observable. These peaks are only observable in a primitive cell and would not be indexed in a body-centered tetragonal space group, e.g. $I \overline{4} 2 \mathrm{~m}$ as proposed recently ${ }^{35}$. We are not the first to measure this; practically every diffractogram of ball-milled "cubic" $\mathrm{Na}_{3} \mathrm{PS}_{4}$ reported in the literature features these reflections $4,5,36,6,7,9,11,14-16,30$. However, this observation has never been adequately explained: it is unclear whether these reflections originate from a secondary tetragonal phase, are indicative of a tetragonal average structure or whether they originate from ordering of the main phase.

Recently, Krauskopf et al. remarked that the local structure of the BM-cub sample is better described by an average tetragonal model, highlighting a discrepancy between average and local structures observable by Bragg- and total scattering diffraction experiments 7 . Our symmetry-confined PDF analysis of a single-unit-cell, shown in Figure $4 \mathrm{~b}$, confirms this observation : the refinement of the local structure of the BM-cub sample from PDF analysis in the $P \overline{4} 2_{1} c$ tetragonal space group $\left(\mathrm{R}_{\mathrm{w}}=0.116\right)$ results in a much better fit of the data compared to those for the cubic $I \overline{4} 3 m$ space group (Table $\mathrm{S}_{5}, \mathrm{R}_{\mathrm{w}}>0.167$ ) for the range 4-20 $\AA$ (see comment regarding the exclusion of first neighbors in the Methods section). We note that in both cases the anisotropy of the $\mathrm{Na}^{+}$density around the $\mathrm{Na}$ position is evident from the PDF fits (structure visualizations in Figure $4 \mathrm{~b}$ ). 
Spectroscopy analysis of local structures. Vibrational spectroscopy experiments were performed to probe the local structure of the $\mathrm{Na}_{3} \mathrm{PS}_{4}$ samples further. The measured Raman spectra of the three $\mathrm{Na}_{3} \mathrm{PS}_{4}$ samples (Figure $5 \mathrm{a}$ ) reveal no differences between the samples, (apart from a distinct fluorescence of the BM-cubic sample which lies outside the scope of this study). At room temperature, the spectra are dominated by the four internal normal modes of the thiophosphate tetrahedral polyanions as indexed in Figure $5 a\left(v_{1} \sim 412, v_{2} \sim 214\right.$, $\left.v_{3} \sim 535, v_{4} \sim 280 \mathrm{~cm}^{-1}\right)$. A distinct splitting of the normal modes is observed, especially in the asymmetric stretching and bending modes, $v_{3}$ and $v_{4}$, respectively. This degeneracy is indicative of deviations from the ideal tetrahedral $\left(\mathrm{T}_{\mathrm{d}}\right)$ symmetry of the $\mathrm{PS}_{4^{3-}}$ moiety and can correlate to the point group symmetry of the phosphorus site in the crystal structure. Indeed, in the cubic $I \overline{4} 3 m$ description, the phosphorus occupies the a Wyckoff position with $-43 \mathrm{~m}\left(\mathrm{~T}_{\mathrm{d}}\right)$ point group symmetry; while, in the tetragonal $P-42_{1} \mathrm{C}$ description, the phosphorus occupies the $2 \mathrm{a}$ Wyckoff with point group symmetry -4 . In other words, the vibrations of the $\mathrm{PS}_{4}{ }^{3-}$ in the tetragonal space-group are slightly distorted due to the absence of the ideal $T_{d}$ point symmetry.

To further rationalize this observation, we simulated the vibrational frequencies/energies for the two polymorphs using DFT, as shown in Figure 5b. The calculations indicate that there should be significant differences between the $\alpha$ - $\left(P \overline{4} 2_{1} c\right)$ and $\beta$ - $(I \overline{4} 3 \mathrm{~m})$ polymorphs in agreement with our experimental observations. If the local structure of the BM-cub sample were truly cubic, singlet peaks should be observed at 535 and $280 \mathrm{~cm}^{-1}$, associated to the $v_{3}$ and $v_{4}$ modes, respectively, and the symmetric P-S stretching mode $\left(v_{1}\right)$ at $412 \mathrm{~cm}^{-1}$ should be appreciably shifted, neither of which is the case. In all spectra, we observe invariantly the splitting of the $\mathrm{PS}_{4}{ }^{3-}$ normal modes indicating the same non-cubic local structure for all samples, including the BM-cub one. These observations are consistent with the PDF interpretation and also in agreement with all reported Raman spectra for ball-milled $\mathrm{Na}_{3} \mathrm{PS}_{4}$ samples in the literature ${ }^{16,37}$. 


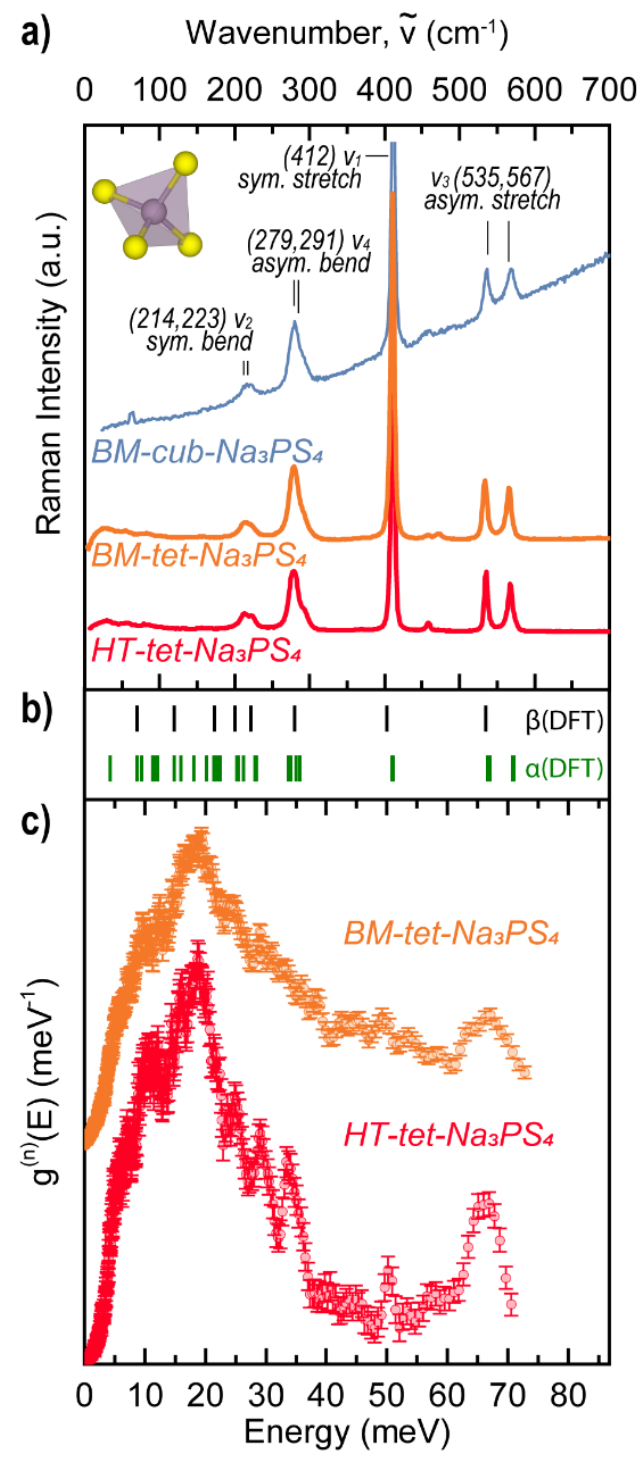

Figure 5 [Single Column]. Phonon spectra of the Na33 $\mathrm{PS}_{4}$ samples. (a) Raman spectra measured at room temperature. (b) DFT-calculated vibrational frequencies/energies based on the published $\alpha$ - and $\beta$-structural models ( $P \overline{4} 2_{1} c$ and $I \overline{4} 3 m$, respectively). (c) Phonon spectra, in terms of generalized density of states (GDOS) extracted from inelastic neutron scattering measurements at room temperature. The $x$-scales in all panels are matched $(E=h c \bar{v})$.

Vibrational modes below $200 \mathrm{~cm}^{-1}(\sim 25 \mathrm{meV})$ are not fundamental but pertain to interactions of the anionic and cationic lattices. Presumably, owing to the high mobility of $\mathrm{Na}^{+}$in $\mathrm{Na}_{3} \mathrm{PS}_{4}$, these modes are broadened and not observable in the Raman spectra at room temperature. However, such modes are well observed in the generalized phonon density of states (GDOS) extracted from inelastic neutron scattering measurements (INS, 
Figure 5c). This is because the INS-measured GDOS involves a weighting of the scattering ions with their scattering power. A distinct broadening of the low energy modes in the GDOS is observed for the ball-milled sample, which can be attributed to the dynamical signature of local mobility and disorder incurred by mechanochemical synthesis. Further, the mode around $65-70 \mathrm{meV}$ of the GDOS, corresponding to the $v_{3}$ assymetric P-S stretch seems to be more separated in the BM-sample and the peak at $\sim 50 \mathrm{meV}$ corresponding to the $v_{1}$ symmetric P-S stretch is significantly dampened. These observations relate to the observed microstrain and high thermal displacement parameters for $\mathrm{P}$ and $\mathrm{S}$ evidenced by diffraction (Tables $\mathrm{S}_{1}-\mathrm{S}_{3}$ ) and could be interpreted to indicate further local deviations of the $\mathrm{PS}_{4}{ }^{3-}$ units from ideality.

The local environment of $\mathrm{Na}$ and $\mathrm{P}$ atoms can be further elucidated through MAS-NMR spectroscopy. The ${ }^{23} \mathrm{Na}$ spectra in Figure 6a show that the sodium ion environments in HTtet- and BM-tet samples have similar sodium local environments with a distinct quadrupolar lineshape, which can be fit using the same quadrupolar NMR parameters with varying line broadening. The fit of the HT-tet spectrum yields two signals with $\delta_{\text {iso }}=1.8$ and $0.9 \mathrm{ppm}, C_{\mathrm{Q}}=2.3(1)$ and 1.7(1) MHz, $\eta=\mathrm{o}$ and o.44, respectively (Figure 6b). While $C_{\mathrm{Q}}$ values are as in line with previous measurements ${ }^{26}$, there is some uncertainty in the fitting of $\delta_{\text {iso }}$ and $\eta$. Similar fits were performed on spectra obtained at two additional field strengths ( $9.4 \& 16.4 \mathrm{~T}$ in addition to $11.8 \mathrm{~T}$ shown here) for HT-tet and show good agreement for the same quadrupolar parameters (Fig. Sio and Table S13). The less distinct features of the BM-tet spectrum are consistent with a greater distribution of quadrupolar environments, as expected from a more disordered sample (smaller crystallite size, higher microstrain, and a distribution of local environments).

The spectrum of BM-cub- $\mathrm{Na}_{3} \mathrm{PS}_{4}$ contains a more symmetrical and narrower resonance with a chemical shift close to the center of mass of the other two spectra. This is consistent with motional averaging and higher Na-ion mobility: Yu et al. ${ }^{11}$ have shown that at lower temperatures a quadrupolar lineshape is still visible for the cubic phase and a transition to a more Gaussian lineshape occurs between $293^{-398} \mathrm{~K}$ or $313^{-3} 33^{\mathrm{K}}$ for static or MAS-NMR 
respectively. The narrower lineshape observed here at lower temperatures is ascribed to a combination of higher mobility and the higher magnetic field strengths used here, i.e. not 9.4 $\mathrm{T}$ but $11.8 \mathrm{~T}$. The latter results in smaller second-order quadrupolar broadening and thus the onset spectral narrowing occurs at lower hop frequencies.

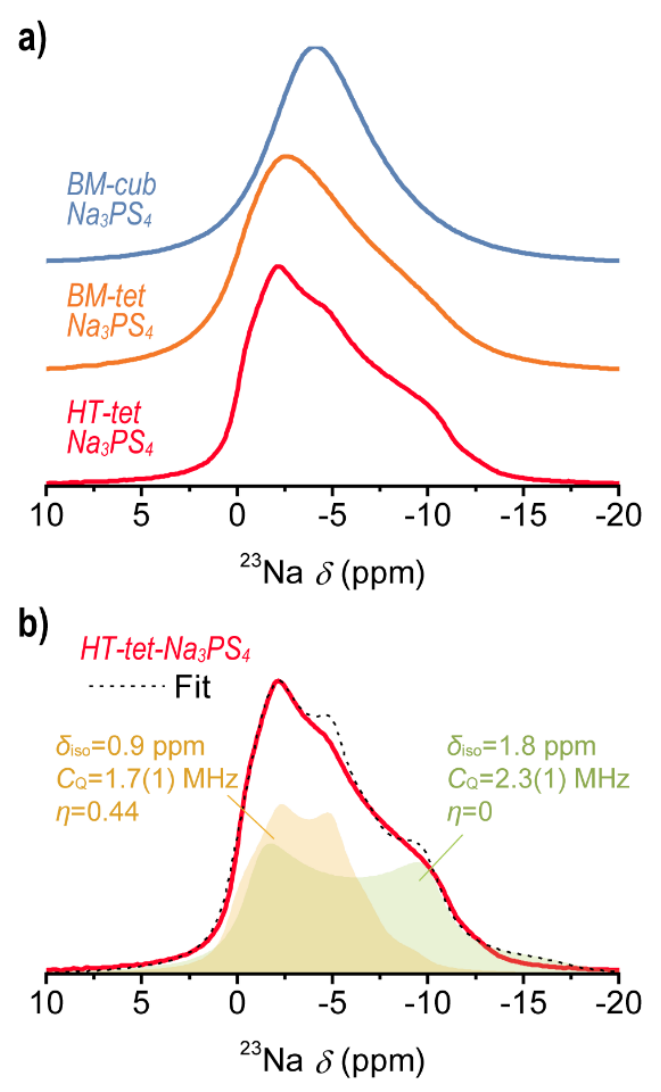

c)

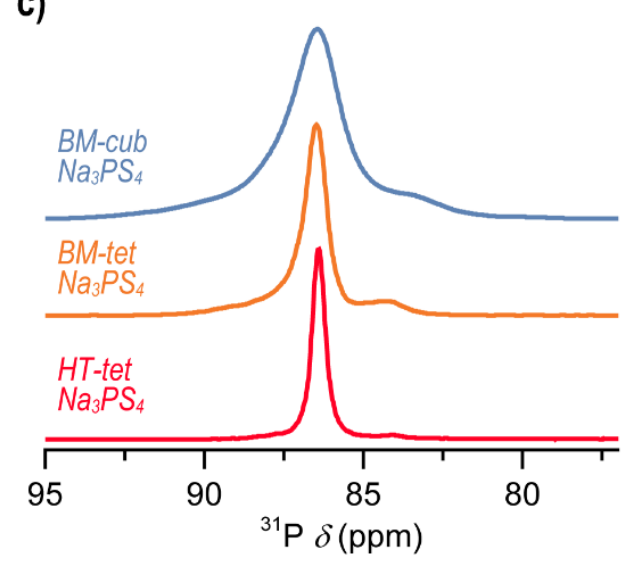

Figure 6 [Single Column]: Room-temperature MAS-NMR spectra of the $\mathrm{Na}_{3} \mathrm{PS}_{4}$ samples. (a) ${ }^{23} \mathrm{Na}$ spectra exhibiting lower quadrupolar broadening and more symmetrical resonance with ballmilling (b) example fit of the ${ }^{23} \mathrm{Na}$ spectrum for HT-tet sample indicating two Na environments. (c) 
${ }^{31} \mathrm{P}$ spectra of the $\mathrm{Na}_{3} \mathrm{PS}_{4}$ samples exhibiting higher broadening of the main $\mathrm{PS}_{4}^{3^{-}}$peak at $\sim 86 \mathrm{ppm}$ with ball-milling.

The characteristic ${ }^{31} \mathrm{P}$ signal for the $\mathrm{PS}_{4}{ }^{3-}$ anion at $\sim 86 \mathrm{ppm}$ shows moderate (BM-tet) or significant (BM-cub) broadening for the ball-milled samples compared to the HT-tet$\mathrm{Na}_{3} \mathrm{PS}_{4}$ sample (Figure 6c), which is consistent with the local disorder and the extensive strain evident from the preceding analysis. The significant broadening for the BM-cub sample indicates much higher disorder and a distribution of local environments causing a wider range of chemical shifts. In order to definitively distinguish between static or dynamic disorder additional variable temperature NMR would be required. The small signal around $84 \mathrm{ppm}$ has previously been assigned to glassy $\mathrm{PS}_{4}{ }^{3-}\left(\& \mathrm{PS}_{3} \mathrm{O}^{-}\right)^{26}$ and there are many possible impurities with a lower shift e.g., various glassy and crystalline phases in the $\mathrm{Na}_{2} \mathrm{~S}-\mathrm{P}_{2} \mathrm{~S}_{5}$ system could cause the resonances of the $\mathrm{PS}_{4}{ }^{3-}$ anions to be shifted to lower frequencies than the $\mathrm{Na}_{3} \mathrm{PS}_{4}$ end member (in analogy to studies in the $\mathrm{Li}_{2} \mathrm{~S}-\mathrm{P}_{2} \mathrm{~S}_{5}$ system ${ }^{38}$ ). Surprisingly, the shift is constant for both tetragonal phases, while the signal shifts to $\sim 83 \mathrm{ppm}$ for the cubic phase suggesting the possibility that peak originates from a defect within the $\mathrm{Na}_{3} \mathrm{PS}_{4}$ and is thus affected by the processing conditions. The higher frequency shoulder is tentatively assigned to connected $\mathrm{PS}_{4}$-tetrahedra, e.g. $\mathrm{P}_{2} \mathrm{~S}_{7^{4-}}$ as similar resonances have been observed in $\mathrm{Li}_{2} \mathrm{~S}_{-} \mathrm{P}_{2} \mathrm{~S}_{5}$ glasses ${ }^{39} . \mathrm{P}_{2} \mathrm{~S}_{7}{ }^{4-}$ units could result, e.g. from $\mathrm{S}^{2-}$ vacancies in $\mathrm{Na}_{3} \mathrm{PS}_{4}$.

The combination of thorough diffraction and spectroscopy analyses on HT- and BM$\mathrm{Na}_{3} \mathrm{PS}_{4}$ converges to the following comparative observations: they possess (i) the same tetragonal local structure (PDF, Raman) irrespective of the synthesis method and (ii) a distinct disorder induced by ball-milling which can be quantified with significantly higher displacement parameters for all atoms (Bragg diffraction, PDF) and which leads to the observation of a more symmetric average structure (Bragg diffraction, NMR). Additionally, the data suggests a rotational disorder of the $\mathrm{PS}_{4}{ }^{3-}$ units (NMR, INS), which are likely statistically distributed in a static manner. 
Variable pressure ionic conductivity and activation volumes. The variation of conductivity with applied pressure is of significant interest from both a fundamental and a practical point of view. Practically, it is widely recognized that applying pressure plays a key role on the electrochemical "cycling" of solid-state batteries and most successful labscale proofs-of-concept utilize custom made setups to effectively measure and control pressure ${ }^{40,41}$. More fundamentally, pressure as a thermodynamic variable can affect the ion dynamics of materials which, in turn, dictate functional properties such as ionic conductivity. In analogy to the activation energy for ion conduction, $E_{a}$, which describes the evolution of ionic conductivity with temperature through an Arrhenius relation (eq. 1), a characteristic activation volume for conduction, $V_{a}$, can describe the evolution of ionic conductivity with pressure ${ }^{42-52}$. The two activation parameters $E_{a}$ and $V_{a}$ are linked in that the activation volume describes the evolution of activation energy with pressure (eq. 2):

$$
\begin{gathered}
E_{a}=k_{B}\left(\frac{\partial \ln (\sigma T)}{\partial T^{-1}}\right)_{P}(1) \\
V_{a}=\frac{d E_{a}}{d P}=k_{B} T\left(\frac{\beta}{3}-\frac{\partial \ln (\sigma)}{\partial P}\right)_{T}
\end{gathered}
$$

where $k_{B}$ is the Boltzmann constant, and $\beta$ is the material compressibility (the inverse of the bulk modulus). The derivation of eq. (2) from transition state theory is shown in the Supporting Information (eqs. S3-S14). Activation volumes are typically reported in $\mathrm{cm}^{3} / \mathrm{mol}$, but can be converted to $\AA^{3}$ through the Avogadro constant to be directly compared with crystallographic volumes. Reported data of activation volumes of inorganic, cation conductors in the literature are summarized in Table 1. Evidently, there is a distinct lack of such data, especially in recent studies dealing with high-performance, room-temperature $\mathrm{Li}^{+}$and $\mathrm{Na}^{+}$conductors aimed at SSB applications. 
Table 1: Values of activation volume for cation conduction in inorganic solid electrolytes in the literature.

\begin{tabular}{|c|c|c|c|}
\hline Compound & $\begin{array}{c}\text { Temperature } \\
\left({ }^{\circ} \mathrm{C}\right)\end{array}$ & $\begin{array}{l}\text { Activation Volume } \\
\left(\mathrm{cm}^{3} / \mathrm{mol}\right)\end{array}$ & Ref. \\
\hline $\operatorname{RbAg}_{4} I_{5}$ & 18 & -0.32 & 42 \\
\hline $\mathrm{Ag}_{3} \mathrm{SBr}$ & 30 & -1.2 & 43 \\
\hline $\mathrm{Ag}_{3} \mathrm{SI}$ & 30 & -2.3 & 43 \\
\hline $\mathrm{Li}-\beta-\mathrm{Al}_{2} \mathrm{O}_{3}$ & 23 & -0.7 & 45 \\
\hline $\mathrm{Na}-\beta-\mathrm{Al}_{2} \mathrm{O}_{3}$ & 23 & $\sim 0$ & 45,46 \\
\hline $\mathrm{K}-\beta-\mathrm{Al}_{2} \mathrm{O}_{3}$ & 23 & 1.3 & 45 \\
\hline$\alpha-A g I$ & 382 & 0.9 & 47 \\
\hline $\mathrm{LiBH}_{4}$ & 220 & 4.6 & 48 \\
\hline $\mathrm{Li}_{0.95} \mathrm{Ca}_{0.025} \mathrm{BH}_{4}$ & 220 & 3.2 & 48 \\
\hline $\mathrm{Li}_{0.35} \mathrm{La}_{0.52} \mathrm{TiO}_{3}$ & -35 to 21 & 1.6 to 1.7 & 49 \\
\hline$\alpha-\mathrm{Li}_{2} \mathrm{SO}_{4}$ & 650 & 0.51 & 50 \\
\hline $\begin{array}{l}\mathrm{Li}_{16-2 x} \mathrm{Zn}_{\mathrm{x}}\left(\mathrm{GeO}_{4}\right)_{4} \\
(\mathrm{x}=1,2)^{*}\end{array}$ & 27 & $0.26,0.58$ & 42 \\
\hline $\begin{array}{l}\mathrm{Li}_{3+\mathrm{x}} \mathrm{Ge}_{\mathrm{x}} \mathrm{V}_{\mathrm{1}-\mathrm{x}} \mathrm{O}_{4} \\
(\mathrm{x}=0.25,0.6,0.72)^{*}\end{array}$ & 27 & $6.00,4.34,4.00$ & 42,51 \\
\hline $\begin{array}{l}\mathrm{Na}_{1+x} \mathrm{Zr}_{2} \mathrm{Si}_{\mathrm{x}} \mathrm{P}_{3-\mathrm{x}} \mathrm{O}_{12} \\
(\mathrm{x}=1.8-2.3)\end{array}$ & 300 & 3.0 to 1.6 & 52 \\
\hline $\mathrm{Na}_{3} \mathrm{SbS}_{4}$ & (RT) & $1.20 \pm 0.08$ & $44 * *$ \\
\hline BM-cub-Na ${ }_{3} \mathrm{PS}_{4}$ & 23 & $2.33 \pm 0.04$ & this work \\
\hline HT-tet- $\mathrm{Na}_{3} \mathrm{PS}_{4}$ & 23 & $1.78 \pm 0.02$ & this work \\
\hline
\end{tabular}

*these materials did not show a monotonic behavior with increasing pressure and the activation volume listed refers to the "low pressure" regime; see cited references.

${ }^{* *}$ extracted in this work from literature data, see Figure S13 and Table S16

The activation volume is often physically interpreted as a volume change of the material required for ion motion 48,53 and can be considered as the difference between the volume needed for ion migration $\left(V_{m}\right)$ and the free volume already available in the structure $\left(V_{\mathrm{f}}\right)$ :

$$
V_{a}=V_{m}-V_{f}(3)
$$


This interpretation is elegantly demonstrated in the example of the beta aluminas 45 (Table 1). For $\mathrm{Na}-\beta-\mathrm{Al}_{2} \mathrm{O}_{3}$ the activation volume is $\sim$, indicating facile $\mathrm{Na}^{+}$conduction through $2 \mathrm{D}$ channels in between the alumina planes $\left(\mathrm{V}_{\mathrm{m}}=\mathrm{V}_{\mathrm{f}}\right)$. For the larger $\mathrm{K}^{+}$and $\mathrm{Rb}^{+}$, the activation volume increases because the alumina planes need to expand to allow the larger alkali ions to migrate $\left(V_{m}>V_{f}\right)$. On the other hand, in $\mathrm{Li}-\beta-\mathrm{Al}_{2} \mathrm{O}_{3}$, the smaller $\mathrm{Li}^{+}$is coordinated more strongly and closer to one of the planes rather than in between. Hence, in order for $\mathrm{Li}^{+}$to get to the migration pathway the planes need to contract, giving rise to a negative activation volume $\left(\mathrm{V}_{\mathrm{m}}<\mathrm{V}_{\mathrm{f}}\right)$. In the framework of this interpretation, the activation volume is characteristic of the migration mechanism. The example of the beta aluminas also demonstrates that a lower activation volume is not always desirable. Even with a negative activation volume, $\mathrm{Li}-\beta-\mathrm{Al}_{2} \mathrm{O}_{3}$ shows a much lower ionic conductivity compared to $\mathrm{Na}-\mathrm{B}_{-}-\mathrm{Al}_{2} \mathrm{O}_{3}{ }^{45}$. Nevertheless, negative activation volume seems to be a characteristic of the superionic $\mathrm{Ag}^{+}$conductors $\mathrm{RbAg}_{4} \mathrm{I}_{5}, \mathrm{Ag}_{3} \mathrm{SI}$ and $\mathrm{Ag}_{3} \mathrm{SBr}$ 43. In that sense, the activation volume should be interpreted in the framework of the specific structure and migration mechanism.

In order to determine the activation volume for $\mathrm{Na}_{3} \mathrm{PS}_{4}$, we performed impedance spectroscopy measurements on pelletized samples as a function of in-situ applied pressure within a uniaxial hydraulic press. Similar to variable-temperature experiments, these measurements lead to a significant evolution of the electrical response, as shown in Figure $7 \mathrm{a}$ and $7 \mathrm{~b}$ for the HT-tet and BM-cub samples. From the impedance spectra we extract the ionic conductivity of the samples (Figure $7 \mathrm{c}$ ) through equivalent circuit fitting. Two critical observations emerge, which can be used to clarify the synthesis-property relationships of $\mathrm{Na}_{3} \mathrm{PS}_{4}$ :

(i) The HT- and BM-synthesized samples differ decisively in their evolution of ionic conductivity with applied pressure, as precisely quantified with activation volumes.

(ii) In contrast to their initially lower conductivity, the HT-tet samples exhibit the same order of magnitude of ionic conductivity as the BM samples $\left(10^{-4} \mathrm{~S} / \mathrm{cm}\right)$ upon pressure application. 


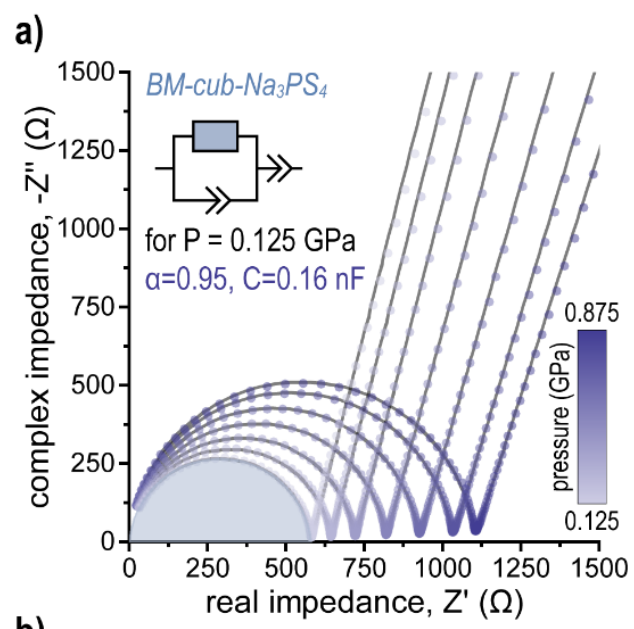

b)
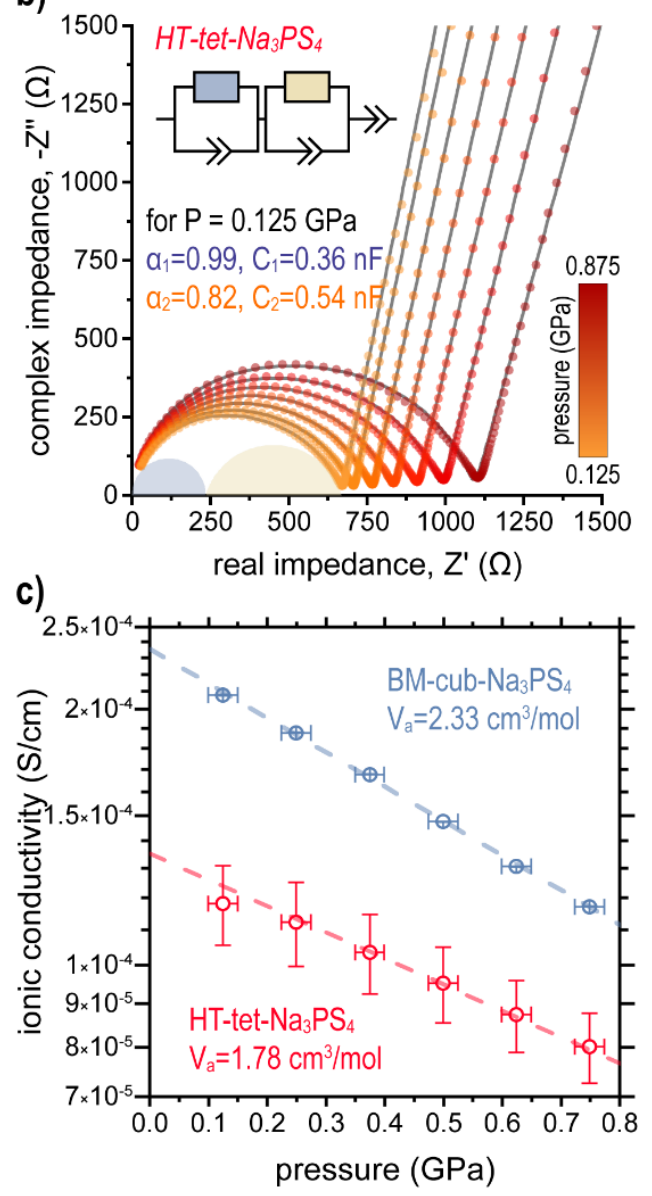

Figure 7 [Single Column]: In-situ variable-pressure impedance spectroscopy experiments. Spectra collected in the direction of pressure release as a function of applied uniaxial pressure in Nyquist coordinates and associated equivalent circuit fits of the HT-tet-Na $\mathrm{PS}_{4}(a)$ and $B M$-cub-Na $\mathrm{PS}_{4}$ (b) samples. (c) Variation of ionic conductivity with pressure for BM-cub-and $\mathrm{HT}$-tet-Na $\mathrm{PS}_{4}$ quantified by their respective activation volumes. 
The impedance spectra of the BM-cub- $\mathrm{Na}_{3} \mathrm{PS}_{4}$ sample can be described with a single diffusion process ( 1 semicircle in Fig. 7a). The extracted capacitance using the Brug relationship ${ }^{54}$ is in the order of $10^{-10} \mathrm{~F}$ and clearly identifies the origin of this conductivity as intragrain ion migration. In contrast, the spectra of the HT-tet- $\mathrm{Na}_{3} \mathrm{PS}_{4}$ sample can be better described by two diffusion processes ( 2 semicircles in Fig. $7 \mathrm{~b}$ ). The two yield significantly different $\omega_{\max }$ by $\sim 1$ order of magnitude but their extracted capacitances are in the same order of magnitude $\left(1 \mathrm{O}^{-10} \mathrm{~F}\right)$. It is unclear at this point if the two can be ascribed to intra- and inter-grain diffusion processes, respectively. We note however, that the respective evolution of the resistances of the two processes show approximately the same evolution with pressure (i.e. activation volume). As such we use their sum in their following analysis to represent the total conductivity of HT-tet- $\mathrm{Na}_{3} \mathrm{PS}_{4}$.

In all cases and noticeably, after initial pressing, the conductivity of $\mathrm{Na}_{3} \mathrm{PS}_{4}$ samples was shown to monotonically decrease with increasing pressure. The evolution of conductivity can be well described with a constant negative slope between the logarithm of conductivity and pressure $\left(\frac{\partial \ln (\sigma)}{\partial P}\right)_{T}$ as shown in Figure $7 \mathrm{c}$. A distinct difference in slope is observable between the BM-cub- and HT-tet- $\mathrm{Na}_{3} \mathrm{PS}_{4}$ samples: the conductivity of the ball-milled sample is more sensitive to the applied pressure. Following equation (2), the observed slope in Figure $7 \mathrm{C}$ was corrected using the compressibility of $\mathrm{Na}_{3} \mathrm{PS}_{4}$ to derive the activation volume. It should be noted that the compressibility of a macroscopic object (pellet) is dependent on its microstructure and especially on the presence of porosity. Unfortunately, our experimental setup did not allow for the in-situ quantification of the density of each sample; however, cold pressing ( $\mathrm{HT}$ or $\mathrm{BM}$ ) $\mathrm{Na}_{3} \mathrm{PS}_{4}$ routinely results in $>95 \%$-dense selfstanding pellets after application of moderate pressures ( $300 \mathrm{MPa})$. As such, in the following analysis, we have assumed that the samples exhibit full densification in the in-situ configuration and as such the same bulk modulus $\mathrm{B} \approx 25 \mathrm{GPa}$ (i.e. $\beta \approx 0.04 \mathrm{GPa}^{-1}$ ), which is an average among our calculations and literature values $55^{-58}$ (Table S14). We note that if densification of the $\mathrm{Na}_{3} \mathrm{PS}_{4}$ powders was still taking place, the exhibited slopes in Figure $7 \mathrm{C}$ should deviate from linearity, and the capacitances derived from fitting the impedance 
spectra (Fig S12) would significantly evolve, neither of which is the case. Under this reasonable assumption, the compressibility correction accounts for $<10 \%$ of the observed variation of ionic conductivity with pressure (Table S15 and Figure S11). We conclude that the activation volume for $\mathrm{Na}^{+}$conduction in the BM sample $\left(2.33 \mathrm{~cm}^{3} / \mathrm{mol}=3.87 \AA^{3}\right)$ is significantly larger (by $\sim 30 \%$ ) than that of the HT sample $\left(1.78 \mathrm{~cm}^{3} / \mathrm{mol}=2.97 \AA^{3}\right)$.

In the $\mathrm{Na}_{3} \mathrm{PS}_{4}$ structure, ion hops occur between the stable lattice sites (6b in the $I \overline{4} 3 \mathrm{~m}$ description of Fig. 1) parallel to the cell edges 13,59 as exemplified in Figure 8 for a jump along the a-direction ([10o]). Between each pair of lattice sites, a four-coordinated site acts as the metastable transition site for ion migration (12d in the $I \overline{4} 3 \mathrm{~m}$ description of Fig. 1). In $\mathrm{Na}_{3} \mathrm{PS}_{4}$, stable lattice sites feature an average $\mathrm{Na}-\mathrm{S}$ bond length of $\sim 2.86 \AA$ corresponding closely to the average $\mathrm{Na}^{\mathrm{IV}}$-S bond of $2.84 \AA$ tabulated by Shannon ${ }^{60}$. In contrast, the average Na-S distance at the transition state is significantly shorter $(\sim 2.65 \AA)$. In order for ion hops to occur, the bottleneck separating the stable site from the transition state needs to expand to allow the $\mathrm{Na}$ ions to transverse it and the observable activation volume can be interpreted as a measure this necessary expansion to allow for ion migration. Applied pressure acts against this necessary expansion increasing the activation energy by $\mathrm{V}_{\mathrm{a}} \cdot \mathrm{P}$ (eq. 2) and thus reducing the ionic conductivity. 


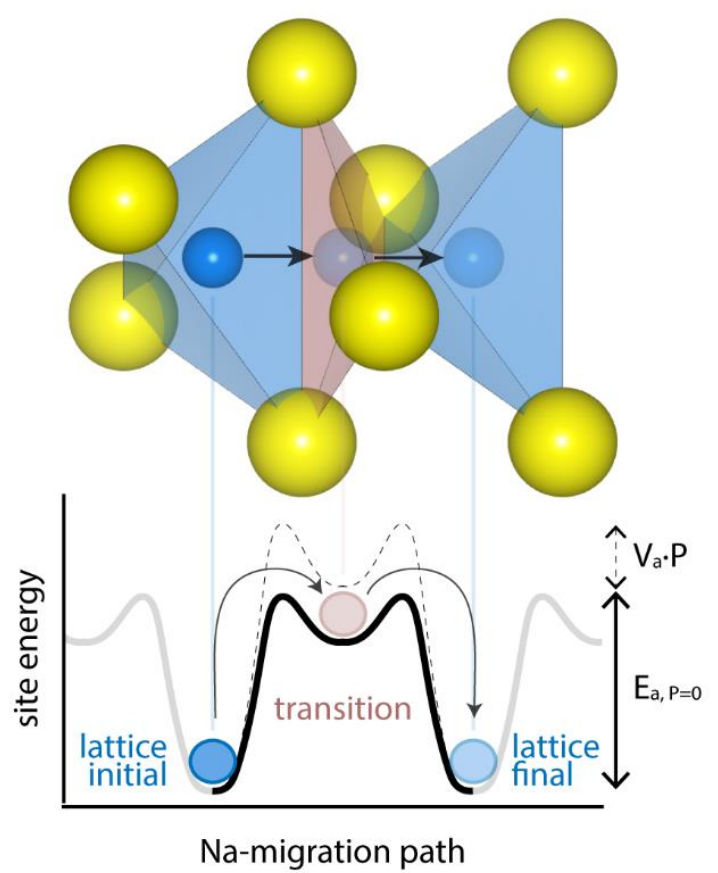

Figure 8 [single column]: Schematic representation of the ion-hopping topology in $\mathrm{Na}_{3} \mathrm{PS}_{4}$ parallel to [100] with associated energy landscape. The transition site (bottleneck) needs to expand to allow for $\mathrm{Na}^{+}$hops between the lattice sites. Applied pressure acts against this expansion by increasing the activation energy for migration, as indicated by the dotted lines.

The significant difference in activation volume between $\mathrm{BM}$-cub- $\mathrm{Na}_{3} \mathrm{PS}_{4}$ and HT-tet- $\mathrm{Na}_{3} \mathrm{PS}_{4}$ can be rationalized on the basis of variations in the available volume for conduction $\left(\mathrm{V}_{\mathrm{f}}\right.$ in eq. 3). The distinct macro- and micro- strain observable from diffraction leads to decreased available volume for $\mathrm{Na}^{+}$to migrate in the structure. The $0.55 \mathrm{~cm} 3 / \mathrm{mol}$ (o.9o $\AA^{3}$ ) difference in activation volume from the variable-pressure impedance measurements can be interpreted as this decrease in available volume for ion conduction owing to the strain incurred by the mechanochemical synthesis method.

It is also interesting to compare our results for the activation volume of $\mathrm{Na}_{3} \mathrm{PS}_{4}$ with results for isostructural compounds. Using the recently reported datasets of Wang et al. for variable-pressure- diffraction and impedance spectroscopy ${ }^{44}$, we derive a bulk modulus of $46 \mathrm{GPa}$ and an activation volume of $1.20 \mathrm{~cm}^{3} / \mathrm{mol}$ for $\mathrm{Na}_{3} \mathrm{SbS}_{4}$ (SI Figure $\mathrm{S}_{3}$ and Table S16). This activation volume is $\sim 25 \%$ lower than that of $\mathrm{HT}$-tet- $\mathrm{Na}_{3} \mathrm{PS}_{4}$. For two isostructural 
materials exhibiting the same conduction mechanism, such as $\mathrm{Na}_{3} \mathrm{PS}_{4}$ and $\mathrm{Na}_{3} \mathrm{SbS}_{4}$, the molar volume of migration, $V_{m}$, can be assumed approximately equal. Substitution of $S b$ in the $\mathrm{Na}_{3} \mathrm{PS}_{4}$ structure causes the unit cell volume to expand by $\sim 9 \%$ (from $\sim 170$ to $\sim$ $185 \AA 3$ /f.u.). Simultaneously, the volume occupied by the $\mathrm{SbS}_{4}{ }^{3-}$ moieties is only $\sim 2 \%$ larger than that of the $\mathrm{PS}_{4}{ }^{3-}$; meaning that the substitution creates significantly more free volume, $V_{f}$, accessible by $\mathrm{Na}^{+}$for diffusion. This interpretation is qualitatively consistent with a lower activation volume for $\mathrm{Na}_{3} \mathrm{SbS}_{4}$, whose lattice would have to expand to a smaller degree to allow $\mathrm{Na}^{+}$diffusion compared to $\mathrm{Na}_{3} \mathrm{PS}_{4}$. Similarly, we expect the larger, isostructural $\mathrm{Na}_{3} \mathrm{PSe}_{4}{ }^{12,61-63}$ and $\mathrm{Na}_{3} \mathrm{SbSe}_{4}{ }^{62,64}$ to present even lower activation volumes.

Figure 9a compares the impedance spectra of the HT-tet- $\mathrm{Na}_{3} \mathrm{PS}_{4}$ sample before and after the variable pressure experiments, showing a $\sim$ ten-fold increase in ionic conductivity. Before the variable-pressure experiments, the pellet of $\mathrm{Na}_{3} \mathrm{PS}_{4}$ (prepared at $\sim 625 \mathrm{MPa}$ ) exhibited a conductivity of $\sim 10^{-5} \mathrm{~S} / \mathrm{cm}$, typical for a high-temperature synthesized sample ${ }^{1,7,1,1,12,15}$. A second resistive component is observable (second semicircle in the Nyquist plot) exhibiting a capacitance in the $10 \mathrm{nF}$ range, which we associate with ion transfer between particles. After the variable-pressure experiments, the electrical signal significantly changes, exhibiting a single ion-transport phenomenon attributable to intragrain diffusion and severely decreased resistance. Similar observations of increased conductivity upon pressing were found from recent variable-pressure conductivity measurements on the isostructural $\mathrm{Na}_{3} \mathrm{SbS}_{4}{ }^{44}$. These observations are analogous to variations of measured ionic conductivity as a function of pellet preparation which has been reported for several solid electrolytes ${ }^{65}{ }^{-67}$, including ball-milled $\mathrm{Na}_{3} \mathrm{PS}_{4}{ }^{56}$. We attribute this to extrinsic effects on macroscopic ionic percolation through the consolidation of the pellet, improving particle-particle and pellet-electrode contact. Figure $9 \mathrm{~b}$ schematically demonstrates this effect: when a solid electrolyte powder is pressed into a pellet, limited particle-particle contact can severely reduce the effective area available for ion transport $\left(\mathrm{A}_{\text {ionic }}\right)$; forcing all ions to pass through limited mesostructural bottlenecks. In addition, 
tortuosity $(\tau)$ is introduced, increasing the effective distance that ions have to transverse between electrodes. Both of the above effects lead to a significant reduction of the observable ionic conductivity. We underline the difference between the proposed consolidation mechanism and "densification", which would refer to significant change in porosity and the overall density of the pellet. In our view, densification cannot explain the experimental observations since pellets of $\mathrm{Na}_{3} \mathrm{PS}_{4}$ prepared under the conditions used here present a $>95 \%$ compactness already before the variable pressure experiments. On the contrary, the increase of particle contact by consolidation can take place without appreciable change in the overall density of the pellet.

a)

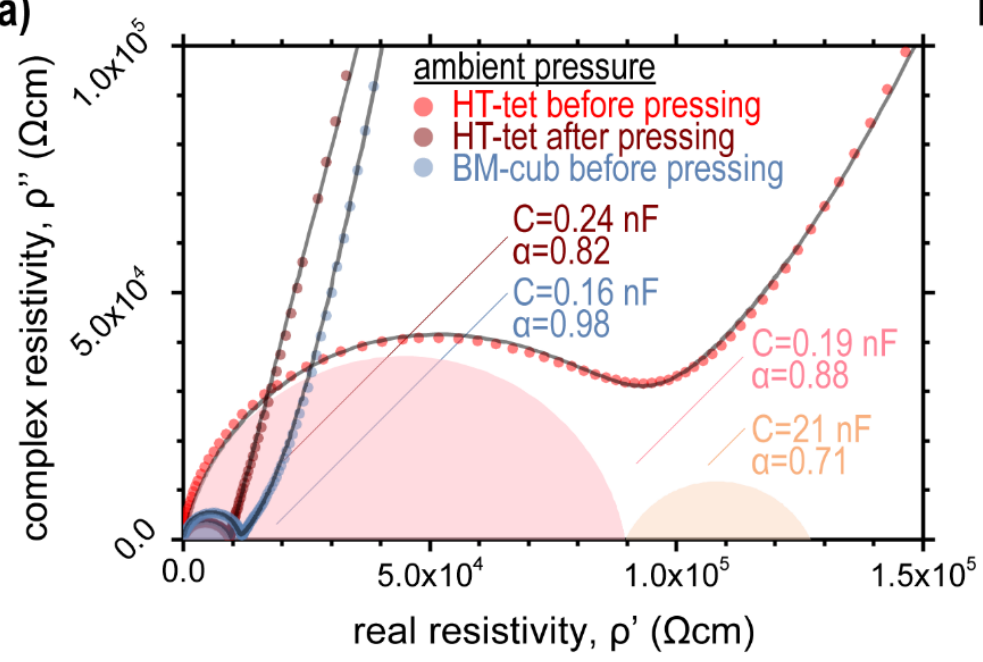

b)

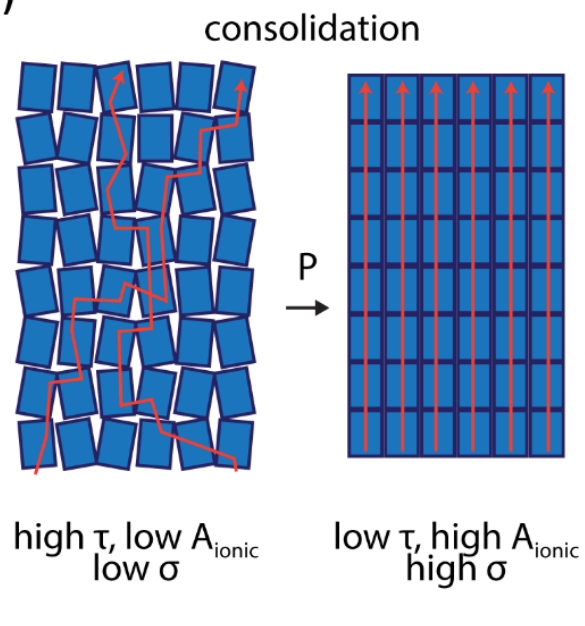

Figure 9 [Double Column]. (a) Comparison of the ambient pressure impedance spectra of HTtet-Na $\mathrm{PS}_{4}$ and BM-cub-Na3 $\mathrm{PS}_{4}$ before and after the variable-pressure experiment in Nyquist coordinates. Data normalized by sample dimensions. (b) Schematic representation of mesoscopic consolidation leading to higher observable conductivity after pressing. Blue rectangles indicate solid electrolyte particles and red lines indicate paths of least resistance for conducting ions.

Furthermore, we highlight that such an effect of consolidation was not evident for the $\mathrm{BM}-\mathrm{Na}_{3} \mathrm{PS}_{4}$ sample. We hypothesize that the significantly smaller particle size of the ballmilled sample allows for its facile densification and thus much easier access to the full potential of the material in terms of macroscopic ionic conductivity. This effect alone can account for the majority of the observed differences in the literature between HT- and BM-synthesized samples, which have been previously ascribed to introduction of mobile 
defects. Nevertheless, the fact that the ball-milled samples are still slightly more conducting (despite their higher activation volume) means that the defect introducing nature of ball-milling cannot be discarded as a parallel influence on the ion transport.

Through our observations on $\mathrm{Na}_{3} \mathrm{PS}_{4}$, our study highlights some limitations of the current commonplace approaches to probing the performance of solid electrolytes. In particular, impedance spectroscopy on pelletized samples largely depends on the procedure of pellet preparation and the conductivities measured should not necessarily reflect the intrinsic behavior of the material on the atomic scale. Impedance spectroscopy experiments performed at low temperatures ${ }^{65,68}$ and/or on single-crystals ${ }^{69,70}$ can distinguish or eliminate the microstructural effects, respectively, thus revealing the "real" intrinsic ion-transport at the atomic scale. Furthermore, we hope to inspire further variable-pressure work to elucidate the effect of common fabrication procedures, relevant to solid-state battery applications. For example, variable-pressure impedance spectroscopy can elucidate the atomistic ion-conduction mechanisms through determination of activation volumes and can lead to identification of processing side-effects, allowing in turn the unlocking of the performance of solid electrolytes, as we have demonstrated here for $\mathrm{Na}_{3} \mathrm{PS}_{4}$.

\section{CONCLUSION}

We have enhanced our current understanding of the effect of mechanochemistry on the structural and ion transport properties of $\mathrm{Na}_{3} \mathrm{PS}_{4}$ through the combined use of diffraction (Bragg and PDF), spectroscopic (impedance, Raman, INS and NMR) and $a b$ initio simulations. We find that ball-milling imparts micro- and macro strain on the material. The local tetragonal structural motif remains unchanged but, the average structure is pseudo-cubic, with disorder introduced in the Na-ion distribution. The stabilization of the cubic polymorph can be understood as a pressure-induced phase transition. Three parallel effects of the mechanochemical synthesis on ion transport at multiple scales are identified: 
(i) Due to the mechanical pressure that acts on the solid electrolyte during the harsh mechanochemical conditions, significant strain is imparted on the material; this strain increases the activation volume, hindering ion transport.

(ii) The repeated impacts during the mechanochemical synthesis decrease the particle size of the resulting powder, facilitating its consolidation into highly ion-conducting pellets.

(iii) Mobile sodium defects are introduced into the $\mathrm{Na}_{3} \mathrm{PS}_{4}$, enhancing ion transport.

While effect (i) hinders ion transport, effects (ii) and (iii) enhance it significantly, leading to an overall increase in ionic conductivity. Indeed, we show that the effect of ballmilling on increasing the ionic conductivity of $\mathrm{Na}_{3} \mathrm{PS}_{4}$ to $\sim 10^{-4} \mathrm{~S} / \mathrm{cm}$ can be reproduced by applying external pressure on a sample from high temperature synthesis.

These results are of importance to the battery community, in which the effects of pressure and (micro-)mechanics on performance have not been fully explored. Indeed, this study aims to inspire further work using variable-pressure diffraction and variable-pressure impedance spectroscopy on solid electrolyte materials.

\section{METHODS}

Synthesis. Three samples were considered in this work, following the work of Krauskopf et al. ${ }^{7}$ and denoted in the same way. The reagents used were the binaries $\mathrm{Na}_{2} \mathrm{~S}$ and $\mathrm{P}_{2} \mathrm{~S}_{5}$ (Sigma-Aldrich) utilized as received.

HT-tetragonal $\mathrm{Na}_{3} \mathrm{PS}_{4}$ was synthesized by a solid-state synthesis route as first reported by Jansen and Henseler ${ }^{1}$. Stoichiometric ratios of the reagents were intimately mixed first in a mortar pestle and then in a ball-mill jar (typically $5 \mathrm{~g}$ of precursors with 25 10-mm $\mathrm{ZrO}_{2}$ balls in an $80 \mathrm{ml} \mathrm{ZrO}_{2}$ jar, milled for $12 \mathrm{~h}$ at $500 \mathrm{rpm}$ ). The resulting powder was pelletized and placed in carbon-coated quartz tubes. The carbon-coating of the tubes was achieved by acetone pyrolysis and subsequent annealing at $1000{ }^{\circ} \mathrm{C}$ for $12 \mathrm{~h}$ under Ar. The quartz tubes were then flame-sealed under vacuum $\left(\sim 10^{-2} \mathrm{mbar}\right)$ and placed in a furnace for reaction. The temperature of the furnace was slowly increased to $500{ }^{\circ} \mathrm{C}$ at $1{ }^{\circ} \mathrm{C} / \mathrm{min}$, held for $20 \mathrm{~h}$ and naturally cooled to room temperature. 
BM-cubic $\mathrm{Na}_{3} \mathrm{PS}_{4}$ was synthesized by a mechanochemical route as first reported by Hayashi et al. ${ }^{4}$. Stoichiometric ratios of the reagents were intimately mixed in a mortar and pestle and placed in an $80 \mathrm{ml} \mathrm{ZrO}$, ball-milling jar along with $60 \mathrm{~g}$ of $3-\mathrm{mm} \mathrm{ZrO}_{2}$ balls. The jars were placed in a planetary ball-mill (Pulverisette 7 Premium, Fritsch) and milled for $12 \mathrm{~h}$ at $500 \mathrm{rpm}$ (144 cycles of 5 minutes of milling followed by 15 -minute breaks to dissipate heat). After the milling treatment the resulting powder was subjected to a heat treatment at $260^{\circ} \mathrm{C}$ under vacuum in a vacuum oven (Büchi).

BM-tetragonal $\mathrm{Na}_{3} \mathrm{PS}_{4}$ was synthesized by a combination of the above methods as first reported by Krauskopf et al.7: a BM-cubic sample was pelletized and sealed in a quartz tube and then placed in an oven preheated to $500{ }^{\circ} \mathrm{C}$ for 20 minutes for a rapid thermal annealing.

In all cases, the products after synthesis were milled by pestle and mortar into fine powders for further analysis. All handling was performed in Ar-filled gloveboxes.

Bragg diffraction. X-ray powder diffraction experiments were performed at the MSPD beamline ${ }^{71,72}$ of the ALBA synchrotron in Barcelona, Spain. Samples were flame-sealed under Ar in 1-mm-diameter quartz capillaries. Diffractograms were collected at room temperature, in transmission geometry, using a position-sensitive MYTHEN detector and a wavelength of $0.8262 \AA$, selected using a Si 111 reflection double crystal monochromator. A well-crystallized $\mathrm{Na}_{2} \mathrm{Ca}_{3} \mathrm{Al}_{2} \mathrm{~F}_{14}$ powder was also measured as standard to extract the instrument resolution and allow for the quantitative analysis of the peak broadening in the $\mathrm{Na}_{3} \mathrm{PS}_{4}$ samples.

Neutron powder diffraction experiments were performed at the $\mathrm{D}_{2} \mathrm{~B}$ beamline of the Institut Laue-Langevin (ILL) in Grenoble, France. Samples ( 2 g) were sealed in cylindrical vanadium containers using indium wire. Diffractograms were collected at room temperature, in transmission geometry, using $128{ }^{3} \mathrm{He}$ counting tubes and a wavelength of $1.594 \AA$ selected using the (335) reflection of $\mathrm{Ge}[115]$ monochromators.

The analysis of the Bragg diffraction data was performed using the programs in the Fullprof software suite ${ }^{73}$. The background was described as a linear interpolation between manually selected points. Le Bail fits were performed to describe the sample broadening using the Thompson-CoxHastings pseudo-Voigt function ${ }^{74}$ (convoluted with the instrumental resolution in the case of $\mathrm{x}$-rays). The apparent crystallite size and apparent microstrain were extracted from the $\mathrm{x}$-ray datasets in accordance with the Scherrer- ${ }^{75}$ and Stokes-Wilson ${ }^{76}$ relations, respectively, as implemented in 
Fullprof ${ }^{73}$. In subsequent Rietveld analyses, occupancies were fixed to the $\mathrm{Na}_{3} \mathrm{PS}_{4}$ stoichiometry and atomic positions and displacement parameters were fitted.

Maximum entropy method (MEM) calculations were performed using Dysnomia ${ }^{77}$. Structure visualizations produced using $\operatorname{VESTA}^{78}$.

Pair distribution function analysis. X-ray powder total scattering diffraction experiments were performed at the 11-ID-B beamline of the Advanced Photon Source at Argonne National Laboratory using high energy $x$-rays $(\lambda=0.21130 \AA)$ for high values of momentum transfer $\left(Q=24.5 \AA^{-1}\right)$. Samples were flame-sealed under Ar in quartz capillaries. One dimensional diffraction data were obtained by integrating the raw $2 \mathrm{D}$ total scattering data in Fit2D. Pair distribution functions (PDFs) were extracted from the background- and Compton scattering-corrected data following Fourier transform using PDFgetX279.

The analysis of the PDFs was performed using the PDFfitz engine as implemented in PDFgui 79. Structural refinements were limited to single unit cells (no supercells) and constrained by the space group symmetry observed in Bragg diffraction $\left(P \overline{4} 2_{1} c\right.$ or $\left.I \overline{4} 3 m\right)$. In agreement with the observation of Krauskopf et al. ${ }^{7}$, the range $1.5-4 \AA$ in the PDFs, which corresponds to the first neighbors P-S, Na-S and S-S, cannot be well-described with the same peak shape as the remaining PDF and was omitted from the refinements. This is probably related to the strong covalent character of the $\mathrm{PS}_{4}{ }^{3-}$ moiety, in contrast with the ionic interactions at higher $\mathrm{r}$. The following parameters were refined in this order: (1) scale factor, (2) lattice parameters, (3) atomic positions, (4) thermal displacement parameters. For fits of the whole PDF (4-70 $\AA$ ) the instrumental resolution parameters $Q_{\text {damp }}$ and $\mathrm{Q}_{\text {broad }}$ were also refined individually at the end of the refinement process.

The quality of each refinement is quantified by the reliability factor weighted R-value, $\mathrm{R}_{w}$, which describes the difference between the experimental data (obs) and the fit (calc) each data point:

$$
\mathrm{R}_{w}=\sqrt{\frac{\sum_{i=1}^{\mathrm{N}} w\left(r_{i}\right)\left[\mathrm{G}_{o b s}\left(r_{i}\right)-\mathrm{G}_{c a l c}\left(r_{i}\right)\right]^{2}}{\sum_{i=1}^{\mathrm{N}} w\left(r_{i}\right) \mathrm{G}_{o b s}^{2}\left(r_{i}\right)}}
$$

with $G_{o b s}$ and $G_{c a l c}$ being the observed and calculated PDF and $w\left(r_{i}\right)$ the weight for each data point.

NMR spectroscopy. ${ }^{23} \mathrm{Na}$ and ${ }^{31} \mathrm{P}$ MAS-NMR spectra were acquired on a Bruker $500 \mathrm{MHz}$ (11.8 T) magnet with Avance III console using a Bruker 2.5 mm HFX MAS probe, a regular zg/one-pulse 
pulse program with $\pi / 2$ and $\pi / 4$ pulses for ${ }^{31} \mathrm{P}$ and ${ }^{23} \mathrm{Na}$ respectively and a MAS frequency of $25 \mathrm{kHz}$. The spectra were externally referenced against solid $\mathrm{NaCl}(7.21 \mathrm{ppm})^{80}$ for ${ }^{23} \mathrm{Na}$ and $\mathrm{NH}_{4} \mathrm{H}_{2} \mathrm{PO}_{4}$ powder (o.8 ppm) for ${ }^{31} \mathrm{P}$. The reference compounds were used for pulse length optimization as well. TopSpin software was used for raw data handling and spectra were fitted using dmfit software ${ }^{81}$ and the Q mas $1 / 2$ model.

Raman spectroscopy. The samples used for Raman spectroscopy were prepared identically to the diffraction samples; i.e. flame-sealed under Ar in 1-mm-diameter quartz capillaries. Spectra were measured using a Renishaw inVia Qontor confocal microscope equipped with a $532 \mathrm{~nm}$ laser excitation source. Spectra were collected in backscattering geometry using a Centrus detector in the range $15-128 \mathrm{o} \mathrm{cm}^{-1}$. Typically, three spectra were recorded and added for a total acquisition time of $3 \times 60=180 \mathrm{~s}$. The incident power of the laser was set to $5 \mathrm{~mW}$ focused by a 1ox objective to a spot of about $10 \mu \mathrm{m}$ on the sample. These parameters were optimized to maximize the signal-over-noise ratio while avoiding excessive local heating and decomposition of the sample evidenced by distortion of the spectra.

Inelastic neutron scattering. Samples ( 2 g) for inelastic neutron scattering (INS) measurements were lightly compacted into pellets and sealed in 10-mm diameter $\mathrm{SiO}_{2}$ ampules under vacuum ( $\left.\sim \mathrm{10}^{-2} \mathrm{mbar}\right)$. The ampules were placed in custom made $\mathrm{Nb}$ holders and subsequently in an evacuated furnace environment. Spectra were measured at room temperature using the coldneutron, time-of-flight, time-focusing, IN6 spectrometer at the Institut Laue-Langevin (ILL) in Grenoble, France. An incident wavelength of 5.12 Å was used, offering an elastic energy resolution of $\sim 0.1 \mathrm{meV}$, as determined from a standard vanadium sample. The vanadium sample was also used to calibrate the detectors. The measurements were carried out in the high-resolution, inelastic time focusing mode. The spectra were collected in the up-scattering, neutron energy-gain mode up to $80 \mathrm{meV}$ in terms of the generalized density of states (GDOS). We note that, in contrast to the vibrational density of states, the INS-measured GDOS involves a weighting of the scatterers (ions) with their scattering powers. Data reduction and treatment, including detector efficiency calibration and background subtraction, were performed using standard ILL tools. Data reduction included measuring an identical empty $\mathrm{SiO}_{2}$ ampule and $\mathrm{Nb}$ container under the same experimental conditions. In the incoherent approximation ${ }^{82}$, the $Q$-averaged, one-phonon ${ }^{83}$ GDOS, $g^{(n)}(E)$, is related to the measured dynamical structure factor, $S(Q, E)$ from INS by: 


$$
g^{(n)}(E)=A\left\langle\frac{e^{2 W(Q)}}{Q^{2}} \frac{E}{n(E, T)+\frac{1}{2} \pm \frac{1}{2}} S(Q, E)\right\rangle
$$

where $A$ is a normalization constant, $2 W(Q)$ is the Debye-Waller factor and $n(E, T)$ is the thermal occupation factor (Bose-factor correction) equal to $\left[\exp \left(E / k_{B} T\right)-1\right]^{-1}$. The + or - signs correspond to neutron energy loss or gain respectively and the brackets indicate an average over all $Q$.

Vibrational spectra calculations. The vibrational spectra of $\mathrm{Na}_{3} \mathrm{PS}_{4}$ were simulated using density functional theory (DFT) as implemented in the all-electron code CRYSTAl $17{ }^{84}$, where the crystalline wavefunction is expanded as a linear combination of atomic orbitals and further expanded by a consistent triple- $\zeta$ plus polarization basis-set ${ }^{85,86}$. The total energy during the geometry relaxation and later in the phonon calculations was converged within $\sim 3.0 \times 10^{-9} \mathrm{eV}$ and integrated over a well converged and symmetrized $8 \times 8 \times 8 k$-point mesh (i.e., the shrinking factor is 8). The unknown exchange-correlation contribution to the total energy was approximated by the range-separated hybrid functional proposed by Heyd-Scuseria-Ernzerhof HSEo6 ${ }^{87,88}$. The truncation of the (infinite) Coulomb and exchange series was set by the tolerances (TOLINTEG): $10^{-7}, 10^{-7}, 10^{-7}, 10^{-9}$ and $10^{-30}$, while an XXLGRID was used for the integration of the charge density. Raman frequencies of the two polymorphs were computed at the $\Gamma$-point only, employing the finite difference method. Thus, the diagonal matrix was developed using a step size of $0.003 \AA$ for each displacement.

Geometry optimization calculations. Ab initio geometry optimization calculations in this work were carried out using DFT with the Vienna ab initio simulation package (VASP) ${ }^{89}$. Plane-wave cut-off energies of 520 were utilized for the geometry optimization calculations. The projector augmented wave method $^{90}$ and the PBEsol exchange-correlation functional ${ }^{91}$ were employed for all calculations. A $k$-point mesh spacing smaller than $0.05 \AA^{-1}$ was used for the geometry optimization calculations.

Impedance spectroscopy. Measurements in temperature were performed on pelletized samples with carbon electrodes. The synthesized $\mathrm{Na}_{3} \mathrm{PS}_{4}$ powders were placed between two 6mm-diameter graphite paper electrodes (Papyex, Mersen) dried overnight under vacuum at $200{ }^{\circ} \mathrm{C}$. The carbon- $\mathrm{Na}_{3} \mathrm{PS}_{4}$-carbon composite pellets were pressed using a 6-mm-diameter steel mold and 
hydraulic press. The pressure was slowly increased in increments of 0.5 tons up to 2 tons (corresponding to $\sim 700 \mathrm{MPa}$ ). In all cases, this resulted in highly dense pellets of $>95 \%$ compacity with respect to the crystallographic density $\left(2.1 \mathrm{~g} / \mathrm{cm}^{3}\right)$. The composite pellets were mounted on an CESH sample holder (Bio-Logic) and placed in an ITS system (Bio-Logic) allowing temperature control in the range of -15 to $100{ }^{\circ} \mathrm{C}$. The spectra collected by applying an AC excitation voltage of $50 \mathrm{mV}$ from $10 \mathrm{~Hz}$ to $30 \mathrm{MHz}$ using an MTZ-35 impedance analyzer (Bio-Logic).

Measurements in pressure were performed on similar samples pressed in custom-made 10-mm-diameter polycarbonate molds and steel pistons. The samples were pressed in an Ar-filled glovebox up to 5 tons $(-625 \mathrm{MPa})$ and protected from air exposure using vacuum grease on the pistons. Typically, 10omg of $\mathrm{Na}_{3} \mathrm{PS}_{4}$ powder was used giving rise to an electrolyte thickness of $\sim 0.6 \mathrm{~mm}$. The ensemble was removed from the glovebox and measurements were performed "in-situ" using the steel pistons as electrodes while controlling the pressure in the range of $0-5$ tons and the same parameters as above. Electrical tape was used to insulate the piston-electrodes from the hydraulic press.

\section{ACKNOWLEDGEMENTS}

The authors are grateful to the Institut Laue-Langevin (ILL) for providing beam time on the $\mathrm{D}_{2} \mathrm{~B}$ diffractometer for the powder diffraction measurements and the IN6 spectrometer for the inelastic neutron scattering (INS) measurements; to the ALBA synchrotron for providing beam time on the MSPD diffractometer for powder diffraction measurements; and to the Argonne National Laboratory (ANL) for providing beam time on the 11-ID-B beamline of the Advanced Photon Source (APS) for the powder total scattering measurement.

T. F. is thankful to the ALISTORE ERI and the German Academic Exchange Service (DAAD) for funding in the form of PhD scholarships. J.A.D. and M.S.I. gratefully acknowledge the EPSRC Programme Grant (EP/Moo9521/1) for funding and the MCC/Archer consortium (EP/Looo202/1) for computational resources. J.A.D. also gratefully acknowledges Newcastle University for funding through a Newcastle Academic Track (NUAcT) Fellowship. P. C. acknowledges funding from the National Research Foundation under his NRFF NRFF12-2020-0012 and the ANR-NRF NRF2019-NRFANRo73 Na-MASTER. This research used resources of the Advanced Photon Source, a U.S. 
Department of Energy (DOE) Office of Science User Facility operated for the DOE Office of Science by Argonne National Laboratory under Contract No. DE-AC02-06CH11357. 


\section{REFERENCES}

(1) Jansen, M.; Henseler, U. Synthesis, Structure Determination, and Ionic Conductivity of Sodium Tetrathiophosphate. J. Solid State Chem. 1992, 99 (1), 110-119. https://doi.org/10.1016/o0224596(92)90295-7.

(2) Famprikis, T.; Dawson, J. A.; Fauth, F.; Clemens, O.; Suard, E.; Fleutot, B.; Courty, M.; Chotard, J.-N.; Islam, M. S.; Masquelier, C. A New Superionic Plastic Polymorph of the $\mathrm{Na}^{+}$Conductor $\mathrm{Na}_{3} \mathrm{PS}_{4}$. ACS Mater. Lett. 2019, 1 (6), 641-646. https://doi.org/10.1021/acsmaterialslett.9boo322.

(3) Famprikis, T.; Canepa, P.; Dawson, J. A.; Islam, M. S.; Masquelier, C. Fundamentals of Inorganic SolidState Electrolytes for Batteries. Nat. Mater. 2019, 18 (12), 1278-1291. https://doi.org/10.1038/s41563-0190431-3.

(4) Hayashi, A.; Noi, K.; Sakuda, A.; Tatsumisago, M. Superionic Glass-Ceramic Electrolytes for RoomTemperature Rechargeable Sodium Batteries. Nat. Commun. 2012, 3 (1), 856. https://doi.org/10.1038/ncomms1843.

(5) Hayashi, A.; Noi, K.; Tanibata, N.; Nagao, M.; Tatsumisago, M. High Sodium Ion Conductivity of Glass-Ceramic Electrolytes with Cubic $\mathrm{Na}_{3} \mathrm{PS}_{4}$. J. Power Sources 2014, 258, 420-423. https://doi.org/10.1016/j.jpowsour.2014.02.054.

(6) Noi, K.; Hayashi, A.; Tatsumisago, M. Structure and Properties of the $\mathrm{Na}_{2} \mathrm{~S}-\mathrm{P}_{2} \mathrm{~S}_{5}$ Glasses and GlassCeramics Prepared by Mechanical Milling. J. Power Sources 2014, 269, 260-265. https://doi.org/10.1016/j.jpowsour.2014.06.158.

(7) Krauskopf, T.; Culver, S. P.; Zeier, W. G. Local Tetragonal Structure of the Cubic Superionic Conductor $\mathrm{Na}_{3} \mathrm{PS}_{4}$. Inorg. Chem. 2018, 57 (8), 4739-4744. https://doi.org/10.1021/acs.inorgchem.8boo458.

(8) Nishimura, S.; Tanibata, N.; Hayashi, A.; Tatsumisago, M.; Yamada, A. The Crystal Structure and Sodium Disorder of High-Temperature Polymorph $\beta-\mathrm{Na}_{3} \mathrm{PS}_{4}$. J. Mater. Chem. A 2017, 5 (47), 2502525030. https://doi.org/10.1039/C7TAo8391B.

(9) Tanibata, N.; Noi, K.; Hayashi, A.; Kitamura, N.; Idemoto, Y.; Tatsumisago, M. X-Ray Crystal Structure Analysis of Sodium-Ion Conductivity in $94 \mathrm{Na}_{3} \mathrm{PS}_{4} \cdot 6 \quad \mathrm{Na}_{4} \mathrm{SiS}_{4}$ Glass-Ceramic Electrolytes. ChemElectroChem 2014, 1 (7), 1130-1132. https://doi.org/10.1002/celc.201402016.

(10) Palatnik, L. S.; Kosevich, V. M.; Tyrina, L. V. Electron Diffraction Examination of the Metastable Phases in the Alloys Au- Sb, In- Sb, In- Bi, and In- Bi-Sb. Russ. Phys. Met. Met. 1961, 11 (2), 75-80.

(11) Yu, C.; Ganapathy, S.; de Klerk, N. J. J.; van Eck, E. R. H.; Wagemaker, M. Na-Ion Dynamics in Tetragonal and Cubic $\mathrm{Na}_{3} \mathrm{PS}_{4}$, a Na-Ion Conductor for Solid State Na-Ion Batteries. J. Mater. Chem. A 2016, 4 (39), 15095-15105. https://doi.org/10.1039/C6TAo5896E.

(12) Bo, S.-H.; Wang, Y.; Ceder, G. Structural and Na-Ion Conduction Characteristics of $\mathrm{Na}_{3} \mathrm{PS}_{\mathrm{x}} \mathrm{Se}_{4-\mathrm{X}}$. J. Mater. Chem. A 2016, 4 (23), 9044-9053. https://doi.org/10.1039/C6TA03027K.

(13) De Klerk, N. J. J.; Wagemaker, M. Diffusion Mechanism of the Sodium-Ion Solid Electrolyte $\mathrm{Na}_{3} \mathrm{PS}_{4}$ and Potential Improvements of Halogen Doping. Chem. Mater. 2016, 28 (9), 3122-3130. https://doi.org/10.1021/acs.chemmater.6boo698.

(14) Hayashi, A.; Tatsumisago, M. All-Solid-State Secondary Cell. US 20170222259 A1.

(15) Takeuchi, S.; Suzuki, K.; Hirayama, M.; Kanno, R. Sodium Superionic Conduction in Tetragonal 
$\mathrm{Na}_{3} \mathrm{PS}_{4}$. J. Solid State Chem. 2018, 265, 353-358. https://doi.org/10.1016/j.jssc.2018.06.023.

(16) Nguyen, H.; Banerjee, A.; Wang, X.; Tan, D.; Wu, E. A.; Doux, J.-M.; Stephens, R.; Verbist, G.; Meng, Y. S. Single-Step Synthesis of Highly Conductive $\mathrm{Na}_{3} \mathrm{PS}_{4}$ Solid Electrolyte for Sodium All Solid-State Batteries. J. Power Sources 2019, 435 (April), 126623. https://doi.org/10.1016/j.jpowsour.2019.05.031.

(17) Kudu, Ö. U.; Famprikis, T.; Fleutot, B.; Braida, M.-D.; Le Mercier, T.; Islam, M. S.; Masquelier, C. A Review of Structural Properties and Synthesis Methods of Solid Electrolyte Materials in the $\mathrm{Li}_{2} \mathrm{~S}-\mathrm{P}_{2} \mathrm{~S}_{5}$ Binary System. J. Power Sources 2018, 407, 31-43. https://doi.org/10.1016/j.jpowsour.2018.10.037.

(18) Breuer, S.; Uitz, M.; Wilkening, H. M. R. Rapid Li Ion Dynamics in the Interfacial Regions of Nanocrystalline Solids. J. Phys. Chem. Lett. 2018, 9 (8), 2093-2097. https://doi.org/10.1021/acs.jpclett.8boo418.

(19) Qiao, A.; Tao, H.; Yue, Y. Enhancing Ionic Conductivity in $\mathrm{Ag}_{3} \mathrm{PS}_{4}$ via Mechanical Amorphization. J. Non. Cryst. Solids 2019, 521 (April), 119476. https://doi.org/10.1016/j.jnoncrysol.2019.119476.

(20) Rao, R. P.; Zhang, X.; Phuah, K. C.; Adams, S. Mechanochemical Synthesis of Fast Sodium Ion Conductor $\mathrm{Na}_{11} \mathrm{Sn}_{2} \mathrm{PSe}_{12}$ Enables First Sodium-Selenium All-Solid-State Battery. J. Mater. Chem. A 2019, 7 (36), 20790-20798. https://doi.org/10.1039/C9TAo6279C.

(21) Schlem, R.; Muy, S.; Prinz, N.; Banik, A.; Shao-Horn, Y.; Zobel, M.; Zeier, W. G. Mechanochemical Synthesis: A Tool to Tune Cation Site Disorder and Ionic Transport Properties of $\mathrm{Li}_{3} \mathrm{MCl}_{6}(\mathrm{M}=\mathrm{Y}, \mathrm{Er})$

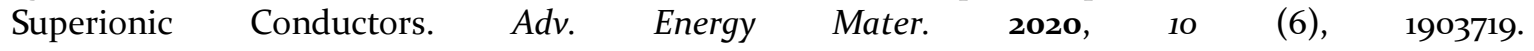
https://doi.org/10.1002/aenm.201903719.

(22) Liu, Z.; Fu, W.; Payzant, E. A.; Yu, X.; Wu, Z.; Dudney, N. J.; Kiggans, J.; Hong, K.; Rondinone, A. J.; Liang, C. Anomalous High Ionic Conductivity of Nanoporous $\beta-\mathrm{Li}_{3} \mathrm{PS}_{4}$. J. Am. Chem. Soc. 2013, 135 (3), 975-978. https://doi.org/10.1021/ja3110895.

(23) Tsukasaki, H.; Mori, S.; Morimoto, H.; Hayashi, A.; Tatsumisago, M. Direct Observation of a NonCrystalline State of $\mathrm{Li}_{2} \mathrm{~S}_{-} \mathrm{P}_{2} \mathrm{~S}_{5} \quad$ Solid Electrolytes. Sci. $\quad$ Rep. $\quad$ 2017, $7 \quad$ (1), 4142. https://doi.org/10.1038/s41598-017-04030-y.

(24) Fuchs, T.; Culver, S. P.; Till, P.; Zeier, W. G. Defect-Mediated Conductivity Enhancements in $\mathrm{Na}_{3-\mathrm{x}} \mathrm{Pn}_{1-}$ ${ }_{\mathrm{x}} \mathrm{W}_{\mathrm{x}} \mathrm{S}_{4}(\mathrm{Pn}=\mathrm{P}, \mathrm{Sb})$ Using Aliovalent Substitutions. ACS Energy Lett. 2020, 5 (1), 146-151. https://doi.org/10.1021/acsenergylett.9bo2537.

(25) Hayashi, A.; Masuzawa, N.; Yubuchi, S.; Tsuji, F.; Hotehama, C.; Sakuda, A.; Tatsumisago, M. A Sodium-Ion Sulfide Solid Electrolyte with Unprecedented Conductivity at Room Temperature. Nat. Commun. 2019, 10 (1). https://doi.org/10.1038/s41467-019-13178-2.

(26) Feng, X.; Chien, P.-H.; Zhu, Z.; Chu, I.-H.; Wang, P.; Immediato-Scuotto, M.; Arabzadeh, H.; Ong, S. P.; Hu, Y.-Y. Studies of Functional Defects for Fast Na-Ion Conduction in $\mathrm{Na}_{3-\mathrm{y}} \mathrm{PS}_{4-\mathrm{x}} \mathrm{Cl}_{\mathrm{x}}$ with a Combined Experimental and Computational Approach. Adv. Funct. Mater. 2019, 29 (9), 1807951. https://doi.org/10.1002/adfm.201807951.

(27) Moon, C. K.; Lee, H.-J.; Park, K. H.; Kwak, H.; Heo, J. W.; Choi, K.; Yang, H.; Kim, M.-S.; Hong, S.-T.; Lee, J. H.; Jung, Y. S. Vacancy-Driven $\mathrm{Na}^{+}$Superionic Conduction in New Ca-Doped $\mathrm{Na}_{3} \mathrm{PS}_{4}$ for AllSolid-State Na-Ion Batteries. ACS Energy Lett. 2018, 3 (10), 2504-2512. https://doi.org/10.1021/acsenergylett.8bo1479.

(28) Chu, I.-H.; Kompella, C. S.; Nguyen, H.; Zhu, Z.; Hy, S.; Deng, Z.; Meng, Y. S.; Ong, S. P. Room- 
Temperature All-Solid-State Rechargeable Sodium-Ion Batteries with a Cl-Doped $\mathrm{Na}_{3} \mathrm{PS}_{4}$ Superionic Conductor. Sci. Rep. 2016, 6 (1), 33733. https://doi.org/10.1038/srep33733.

(29) Zhu, Z.; Chu, I.-H.; Deng, Z.; Ong, S. P. Role of $\mathrm{Na}^{+}$Interstitials and Dopants in Enhancing the $\mathrm{Na}^{+}$ Conductivity of the Cubic $\mathrm{Na}_{3} \mathrm{PS}_{4}$ Superionic Conductor. Chem. Mater. 2015, 27 (24), 8318-8325. https://doi.org/10.1021/acs.chemmater.5bo3656.

(30) Tanibata, N.; Noi, K.; Hayashi, A.; Tatsumisago, M. Preparation and Characterization of Highly Sodium Ion Conducting $\mathrm{Na}_{3} \mathrm{PS}_{4}-\mathrm{Na}_{4} \mathrm{SiS}_{4}$ Solid Electrolytes. RSC Adv. 2014, 4 (33), 17120-17123. https://doi.org/10.1039/c4raoo996g.

(31) Dawson, J. A.; Canepa, P.; Clarke, M. J.; Famprikis, T.; Ghosh, D.; Islam, M. S. Toward Understanding the Different Influences of Grain Boundaries on Ion Transport in Sulfide and Oxide Solid Electrolytes. Chem. Mater. 2019, 31 (14), 5296-5304. https://doi.org/10.1021/acs.chemmater.9bo1794.

(32) Takacs, L. Self-Sustaining Reactions Induced by Ball Milling. Prog. Mater. Sci. 2oo2, 47 (4), 355-414. https://doi.org/10.1016/Soo79-6425(o1)oooo2-o.

(33) Burmeister, C. F.; Kwade, A. Process Engineering with Planetary Ball Mills. Chem. Soc. Rev. 2013, 42 (18), 766o. https://doi.org/10.1039/c3cs35455e.

(34) Gotor, F. J.; Achimovicova, M.; Real, C.; Balaz, P. Influence of the Milling Parameters on the Mechanical Work Intensity in Planetary Mills. Powder Technol. 2013, 233, 1-7. https://doi.org/10.1016/j.powtec.2012.08.031.

(35) Seidel, S.; Zeier, W. G.; Pöttgen, R. The Polymorphs of the $\mathrm{Na}^{+}$Ion Conductor $\mathrm{Na}_{3} \mathrm{PS}_{4}$ Viewed from the Perspective of a Group-Subgroup Scheme. Zeitschrift für Krist. - Cryst. Mater. 2020, 235 (1-2), 1-6. https://doi.org/10.1515/zkri-2019-0053.

(36) Krauskopf, T.; Pompe, C.; Kraft, M. A.; Zeier, W. G. Influence of Lattice Dynamics on $\mathrm{Na}^{+}$Transport in the Solid Electrolyte $\mathrm{Na}_{3} \mathrm{PS}_{4-\mathrm{x}} \mathrm{Se}$. Chem. Mater. 2017, 29 (20), 8859-8869. https://doi.org/10.1021/acs.chemmater.7bo3474.

(37) Tanibata, N.; Deguchi, M.; Hayashi, A.; Tatsumisago, M. All-Solid-State Na/S Batteries with a $\mathrm{Na}_{3} \mathrm{PS}_{4}$ Electrolyte Operating at Room Temperature. Chem. Mater. 2017, 29 (12), 5232-5238. https://doi.org/10.1021/acs.chemmater.7bo116.

(38) Eckert, H.; Zhang, Z.; Kennedy, J. H. Structural Transformation of Non-Oxide Chalcogenide Glasses. The Short-Range Order of $\mathrm{Li}_{2} \mathrm{~S}_{-} \mathrm{P}_{2} \mathrm{~S}_{5}$ Glasses Studied by Quantitative ${ }^{31}$ and ${ }^{6,7} \mathrm{Li}$ High-Resolution SolidState NMR. Chem. Mater. 1990, 2 (3), 273-279. https://doi.org/10.1021/cmoooogao17.

(39) Dietrich, C.; Weber, D. A.; Sedlmaier, S. J.; Indris, S.; Culver, S. P.; Walter, D.; Janek, J.; Zeier, W. G. Lithium Ion Conductivity in $\mathrm{Li}_{2} \mathrm{~S}-\mathrm{P}_{2} \mathrm{~S}_{5}$ Glasses - Building Units and Local Structure Evolution during the Crystallization of Superionic Conductors $\mathrm{Li}_{3} \mathrm{PS}_{4}, \mathrm{Li}_{7} \mathrm{P}_{3} \mathrm{~S}_{11}$ and $\mathrm{Li}_{4} \mathrm{P}_{2} \mathrm{~S}_{7}$. J. Mater. Chem. A 2017, 5 (34), 18111-18119. https://doi.org/10.1039/C7TAo6o67J.

(40) Zhang, W.; Schröder, D.; Arlt, T.; Manke, I.; Koerver, R.; Pinedo, R.; Weber, D. A.; Sann, J.; Zeier, W. G.; Janek, J. (Electro)Chemical Expansion during Cycling: Monitoring the Pressure Changes in Operating Solid-State Lithium Batteries. J. Mater. Chem. A 2017, 5 (20), 9929-9936. https://doi.org/10.1039/C7TA02730C.

(41) Wu, X.; El Kazzi, M.; Villevieille, C. Surface and Morphological Investigation of the Electrode/Electrolyte Properties in an All-Solid-State Battery Using a $\mathrm{Li}_{2} \mathrm{~S}-\mathrm{P}_{2} \mathrm{~S}_{5}$ Solid Electrolyte. J. 
Electroceramics 2017, 38 (2-4), 207-214. https://doi.org/10.1007/s10832-017-0084-z.

(42) Kim, K. S.; Paik, W.-K. Effects of Temperature and Pressure on Conductance of Solid Electrolyte, $\mathrm{RbAg}_{4} \mathrm{I}_{5}$. J. Chem. Eng. Data 1975, 20 (4), 356-359. https://doi.org/10.1021/je6oo67ao21.

(43) Hoshino, H.; Yanagiya, H.; Shimoji, M. Effect of Hydrostatic Pressure on the Electrical Conductivity of $\mathrm{Ag}_{3} \mathrm{SBr}$ and $\beta-\mathrm{Ag}_{3} \mathrm{SI}$. J. Chem. Soc. Faraday Trans. 1 Phys. Chem. Condens. Phases 1974, 70, 281. https://doi.org/10.1039/fig747000281.

(44) Wang, H.; Yu, M.; Wang, Y.; Feng, Z.; Wang, Y.; Lü, X.; Zhu, J.; Ren, Y.; Liang, C. In-Situ Investigation of Pressure Effect on Structural Evolution and Conductivity of $\mathrm{Na}_{3} \mathrm{SbS}_{4}$ Superionic Conductor. J. Power Sources 2018, 401 (April), 111-116. https://doi.org/10.1016/j.jpowsour.2018.05.037.

(45) Radzilowski, R. H.; Kummer, J. T. The Hydrostatic Pressure Dependence of the Ionic Conductivity of ß-Aluminas. J. Electrochem. Soc. 1971, 118 (5), 714. https://doi.org/10.1149/1.2408152.

(46) Itoh, K.; Kondo, K.; Sawaoka, A.; Saito, S. Effect of Pressure on the Ionic Conduction of Na- $\beta$-Alumina. Jpn. J. Appl. Phys. 1975, 14 (8), 1237-1238. https://doi.org/10.1143/JJAP.14.1237.

(47) Wagener, K. Die Druckabhängigkeit Der Ionenleitfähigkeit Anomaler Mischkristalle von AgBr Und AgJ. Zeitschrift für Phys. Chemie 196o, 23 (5_6), 305-312. https://doi.org/10.1524/zpch.1960.23.5_6.305.

(48) Mezaki, T.; Kuronuma, Y.; Oikawa, I.; Kamegawa, A.; Takamura, H. Li-Ion Conductivity and Phase Stability of Ca-Doped $\mathrm{LiBH}_{4}$ under High Pressure. Inorg. Chem. 2016, 55 (20), 10484-10489. https://doi.org/10.1021/acs.inorgchem.6bo1678.

(49) Inaguma, Y.; Yu, J.; Shan, Y. J.; Itoh, M.; Nakamura, T. The Effect of the Hydrostatic Pressure on the Ionic Conductivity in a Perovskite Lanthanum Lithium Titanate. J. Electrochem. Soc. 1995, 142 (1), 142145. https://doi.org/10.1149/1.2043988.

(50) Mellander, B.-E.; Lazarus, D. Electrical Conductivity and Activation Volume for $\alpha-\mathrm{Li}_{2} \mathrm{SO}_{4}$. Phys. Rev. B 1985, 31 (10), 6801-6803. https://doi.org/10.1103/PhysRevB.31.6801.

(51) Mazumdar, D.; Bose, D. N.; Parthasarathy, G.; Gopal, E. S. R. High-Pressure Studies on Lithium FastIon Conductors. J. Mater. Res. 1987, 2 (3), 366-373. https://doi.org/10.1557/JMR.1987.0366.

(52) Kafalas, J. A.; Cava, R. J. Abstract. In Fast Ion Transport in Solidsı; Vashishta, P., Mundy, J. N., Shenoy, G. K., Eds.; Elsevier: Amsterdam, 1979; p 149.

(53) Fontanella, J. J. Pressure and Temperature Variation of the Electrical Conductivity of Poly(Propylene Glycol) Containing $\mathrm{LiCF}_{3} \mathrm{SO}_{3}$. J. Chem. Phys. 1999, 111 (15), 7103-7109. https://doi.org/10.1063/1.480oo2.

(54) Brug, G. J.; van den Eeden, A. L. G.; Sluyters-Rehbach, M.; Sluyters, J. H. The Analysis of Electrode Impedances Complicated by the Presence of a Constant Phase Element. J. Electroanal. Chem. 1984, 176 (1-2), 275-295. https://doi.org/10.1016/Soo22-0728(84)80324-1.

(55) Deng, Z.; Wang, Z.; Chu, I.-H.; Luo, J.; Ong, S. P. Elastic Properties of Alkali Superionic Conductor Electrolytes from First Principles Calculations. J. Electrochem. Soc. 2016, 163 (2), A67-A74. https://doi.org/10.1149/2.0061602jes.

(56) Nose, M.; Kato, A.; Sakuda, A.; Hayashi, A.; Tatsumisago, M. Evaluation of Mechanical Properties of $\mathrm{Na}_{2} \mathrm{~S}_{-} \mathrm{P}_{2} \mathrm{~S}_{5}$ Sulfide Glass Electrolytes. J. Mater. Chem. A 2015, 3 (44), 22061-22065. https://doi.org/10.1039/C5TAo5590C. 
(57) Liu, W.; Sun, H.; Niu, Y. Theoretical Investigation the Mechanical and Thermodynamic Properties of $\alpha$ and $\beta$-Phase Solid Electrolytes $\mathrm{Na}_{3} \mathrm{PS}_{4}$. J. Electrochem. Soc. 2019, 166 (13), A3011-A3018. https://doi.org/10.1149/2.1081913jes.

(58) Yu, Z.; Shang, S.-L.; Seo, J.-H.; Wang, D.; Luo, X.; Huang, Q.; Chen, S.; Lu, J.; Li, X.; Liu, Z.-K.; Wang, D. Exceptionally High Ionic Conductivity in $\mathrm{Na}_{3} \mathrm{P} 0.62$ As $0.38 \mathrm{~S} 4$ with Improved Moisture Stability for Solid-State Sodium-Ion Batteries. Adv. Mater. 2017, $29 \quad$ (16), 1605561. https://doi.org/10.1002/adma.201605561.

（59） Rush, L. E.; Hood, Z. D.; Holzwarth, N. A. W. Unraveling the Electrolyte Properties of $\mathrm{Na}_{3} \mathrm{SbS}_{4}$ through Computation and Experiment. Phys. Rev. Mater. 2017, 1 (7), 075405. https://doi.org/10.1103/PhysRevMaterials.1.075405.

(6o) Shannon, R. D. Revised Effective Ionic Radii and Systematic Studies of Interatomic Distances in Halides and Chalcogenides. Acta Crystallogr. Sect. A 1976, 32 (5), 751-767. https://doi.org/10.1107/So567739476001551.

(61) Zhang, L.; Yang, K.; Mi, J.; Lu, L.; Zhao, L.; Wang, L.; Li, Y.; Zeng, H. Na ${ }_{3} \mathrm{PSe}_{4}$ : A Novel Chalcogenide Solid Electrolyte with High Ionic Conductivity. Adv. Energy Mater. 2015, 5 (24), 2-6. https://doi.org/10.1002/aenm.201501294.

(62) Wang, N.; Yang, K.; Zhang, L.; Yan, X.; Wang, L.; Xu, B. Improvement in Ion Transport in $\mathrm{Na}_{3} \mathrm{PSe}_{4}{ }^{-}$ $\mathrm{Na}_{3} \mathrm{SbSe}_{4}$ by Sb Substitution. J. Mater. Sci. 2018, 53 (3), 1987-1994. https://doi.org/10.1007/s10853-0171618-0.

(63) Krauskopf, T.; Muy, S.; Culver, S. P.; Ohno, S.; Delaire, O.; Shao-Horn, Y.; Zeier, W. G. Comparing the Descriptors for Investigating the Influence of Lattice Dynamics on Ionic Transport Using the Superionic Conductor $\mathrm{Na}_{3} \mathrm{PS}_{4-\mathrm{x}} \mathrm{Sex} . \quad J$. Am. Chem. Soc. 2018, 140 (43), 14464-14473. https://doi.org/10.1021/jacs.8bo9340.

(64) Xiong, S.; Liu, Z.; Rong, H.; Wang, H.; McDaniel, M.; Chen, H. $\mathrm{Na}_{3} \mathrm{SbSe}_{4-\mathrm{x}} \mathrm{S}_{\mathrm{x}}$ as Sodium Superionic Conductors. Sci. Rep. 2018, 8 (1), 9146. https://doi.org/10.1038/s41598-018-27301-8.

(65) Ohno, S.; Bernges, T.; Buchheim, J.; Duchardt, M.; Hatz, A.-K.; Kraft, M. A.; Kwak, H.; Santhosha, A. L.; Liu, Z.; Minafra, N.; Tsuji, F.; Sakuda, A.; Schlem, R.; Xiong, S.; Zhang, Z.; Adelhelm, P.; Chen, H.; Hayashi, A.; Jung, Y. S.; Lotsch, B. V.; Roling, B.; Vargas-Barbosa, N. M.; Zeier, W. G. How Certain Are the Reported Ionic Conductivities of Thiophosphate-Based Solid Electrolytes? An Interlaboratory Study. ACS Energy Lett. 2020, 5 (3), 910-915. https://doi.org/10.1021/acsenergylett.9bo2764.

(66) Garcia-Mendez, R.; Smith, J. G.; Neuefeind, J. C.; Siegel, D. J.; Sakamoto, J. Correlating Macro and Atomic Structure with Elastic Properties and Ionic Transport of Glassy $\mathrm{Li}_{2} \mathrm{~S}_{-} \mathrm{P}_{2} \mathrm{~S}_{5}$ (LPS) Solid Electrolyte for Solid-State Li Metal Batteries. Adv. Energy Mater. 2020, 10 (19), 2000335. https://doi.org/10.1002/aenm.202000335.

(67) Hayashi, A.; Masuzawa, N.; Yubuchi, S.; Tsuji, F.; Hotehama, C.; Sakuda, A.; Tatsumisago, M. A Sodium-Ion Sulfide Solid Electrolyte with Unprecedented Conductivity at Room Temperature. Nat. Commun. 2019, 10 (1), 5266. https://doi.org/10.1038/s41467-019-13178-2.

(68) Lunghammer, S.; Ma, Q.; Rettenwander, D.; Hanzu, I.; Tietz, F.; Wilkening, H. M. R. Bulk and GrainBoundary Ionic Conductivity in Sodium Zirconophosphosilicate $\mathrm{Na}_{3} \mathrm{Zr}_{2}\left(\mathrm{SiO}_{4}\right)_{2} \mathrm{PO}_{4}$ (NASICON). Chem. Phys. Lett. 2018, 701, 147-150. https://doi.org/10.1016/j.cplett.2018.04.037.

(69) Rettenwander, D.; Welzl, A.; Pristat, S.; Tietz, F.; Taibl, S.; Redhammer, G. J.; Fleig, J. A Microcontact 
Impedance Study on NASICON-Type $\mathrm{Li}_{1+\mathrm{x}} \mathrm{Al}_{\mathrm{x}} \mathrm{Ti}_{2-\mathrm{x}}\left(\mathrm{PO}_{4}\right)_{3}(\mathrm{o} \leq \mathrm{x} \leq 0.5)$ Single Crystals. J. Mater. Chem. A 2016, 4 (4), 1506-1513. https://doi.org/10.1039/C5TAo8545D.

(7o) Iwasaki, R.; Hori, S.; Kanno, R.; Yajima, T.; Hirai, D.; Kato, Y.; Hiroi, Z. Weak Anisotropic Lithium-Ion Conductivity in Single Crystals of $\mathrm{Li}_{10} \mathrm{GeP}_{2} \mathrm{~S}_{12}$. Chem. Mater. 2019, 31 (10), 3694-3699. https://doi.org/10.1021/acs.chemmater.9boo420.

(71) Fauth, F.; Peral, I.; Popescu, C.; Knapp, M. The New Material Science Powder Diffraction Beamline at $\begin{array}{lllllll}\text { ALBA Synchrotron. Powder Diffr. 2013, } & 28 & \text { (S2), }\end{array}$ https://doi.org/10.1017/So88571561300090o.

(72) Fauth, F.; Boer, R.; Gil-Ortiz, F.; Popescu, C.; Vallcorba, O.; Peral, I.; Fullà, D.; Benach, J.; Juanhuix, J. The Crystallography Stations at the Alba Synchrotron. Eur. Phys. J. Plus 2015, 130 (8), 160. https://doi.org/10.1140/epjp/i2015-1516o-y.

(73) Rodríquez-Carvajal, J.; Roisnel, T. Line Broadening Analysis Using FullProf*: Determination of Microstructural Properties. Mater. Sci. Forum 2004, 443-444, 123-126. https://doi.org/10.4028/www.scientific.net/MSF.443-444.123.

(74) Thompson, P.; Cox, D. E.; Hastings, J. B. Rietveld Refinement of Debye-Scherrer Synchrotron X-Ray Data from $\mathrm{Al}_{2} \mathrm{O}_{3}$. J. Appl. Crystallogr. 1987, 20 (2), 79-83. https://doi.org/10.1107/Soo2188988708709o.

(75) Patterson, A. L. The Scherrer Formula for X-Ray Particle Size Determination. Phys. Rev. 1939, 56 (10), 978-982. https://doi.org/10.1103/PhysRev.56.978.

(76) Stokes, A. R.; Wilson, A. J. C. The Diffraction of x Rays by Distorted Crystal Aggregates - I. Proc. Phys. Soc. 1944, 56 (3), 174-181. https://doi.org/10.1088/0959-5309/56/3/303.

(77) Momma, K.; Ikeda, T.; Belik, A. A.; Izumi, F. Dysnomia, a Computer Program for Maximum-Entropy Method (MEM) Analysis and Its Performance in the MEM-Based Pattern Fitting. Powder Diffr. 2013, 28 (3), 184-193. https://doi.org/10.1017/So88571561300002X.

(78) Momma, K.; Izumi, F. VESTA 3 for Three-Dimensional Visualization of Crystal, Volumetric and Morphology Data. J. Appl. Crystallogr. 2011, $44 \quad$ (6), 1272-1276. https://doi.org/doi:10.1107/Soo21889811038970.

(79) Farrow, C. L.; Juhas, P.; Liu, J. W.; Bryndin, D.; Boin, E. S.; Bloch, J.; Proffen, T.; Billinge, S. J. L. PDFfit2 and PDFgui: Computer Programs for Studying Nanostructure in Crystals. J. Phys. Condens. Matter 2007, 19 (33). https://doi.org/10.1088/0953-8984/19/33/335219.

(8o) Hayashi, S.; Hayamizu, K. Shift References in High-Resolution Solid-State NMR. Bulletin of the Chemical Society of Japan. 1989, pp 2429-2430. https://doi.org/10.1246/bcsj.62.2429.

(81) Massiot, D.; Fayon, F.; Capron, M.; King, I.; Le Calvé, S.; Alonso, B.; Durand, J. O.; Bujoli, B.; Gan, Z.; Hoatson, G. Modelling One- and Two-Dimensional Solid-State NMR Spectra. Magn. Reson. Chem. 2002, 40 (1), 70-76. https://doi.org/10.1002/mrc.984.

(82) Skold, K.; Price, D. Neutron Scattering, Volume 23A; Academic Press, 1986.

(83) Sjolander, A. Multi-Phonon Processes in Slow Neutron Scattering by Crystals. Ark. Fys. 1958, 14.

(84) Dovesi, R.; Erba, A.; Orlando, R.; Zicovich-Wilson, C. M.; Civalleri, B.; Maschio, L.; Rérat, M.; Casassa, S.; Baima, J.; Salustro, S.; Kirtman, B. Quantum-Mechanical Condensed Matter Simulations with 
CRYSTAL. Wiley Interdiscip. Rev. Comput. Mol. Sci. 2018, 8 (4), e1360. https://doi.org/10.1002/wcms.136o.

(85) Laun, J.; Vilela Oliveira, D.; Bredow, T. Consistent Gaussian Basis Sets of Double- and Triple-Zeta Valence with Polarization Quality of the Fifth Period for Solid-State Calculations. J. Comput. Chem. 2018, 39 (19), 1285-1290. https://doi.org/10.1002/jcc.25195.

(86) Peintinger, M. F.; Oliveira, D. V.; Bredow, T. Consistent Gaussian Basis Sets of Triple-Zeta Valence with Polarization Quality for Solid-State Calculations. J. Comput. Chem. 2013, 34 (6), 451-459. https://doi.org/10.1002/jcc.23153.

(87) Heyd, J.; Scuseria, G. E.; Ernzerhof, M. Hybrid Functionals Based on a Screened Coulomb Potential. J. Chem. Phys. 2003, 118 (18), 8207-8215. https://doi.org/10.1063/1.156406o.

(88) Krukau, A. V.; Vydrov, O. A.; Izmaylov, A. F.; Scuseria, G. E. Influence of the Exchange Screening Parameter on the Performance of Screened Hybrid Functionals. J. Chem. Phys. 2006, 125 (22), 224106. https://doi.org/10.1063/1.2404663.

(89) Kresse, G.; Furthmüller, J. Efficient Iterative Schemes for Ab Initio Total-Energy Calculations Using a Plane-Wave Basis Set. Phys. Rev. B 1996, 54 (16), 11169-11186. https://doi.org/10.1103/PhysRevB.54.11169.

(9o) Blöchl, P. E. Projector Augmented-Wave Method. Phys. Rev. B 1994, 50 (24), 17953-17979. https://doi.org/10.1103/PhysRevB.50.17953.

(91) Perdew, J. P.; Ruzsinszky, A.; Csonka, G. I.; Vydrov, O. A.; Scuseria, G. E.; Constantin, L. A.; Zhou, X.; Burke, K. Restoring the Density-Gradient Expansion for Exchange in Solids and Surfaces. Phys. Rev. Lett. 2008, 100 (13), 136406. https://doi.org/10.1103/PhysRevLett.100.136406. 